\title{
STABILITY OF CNOIDAL WAVES TO HIROTA-SATSUMA SYSTEMS
}

\author{
Jaime Angulo Pava*
}

Dedicated to Hebe Biagioni, colleague, mentor and friend

\begin{abstract}
This work is concerned with non-linear stability properties of periodic travelling waves solutions of the Hirota-Satsuma systems

$$
\left\{\begin{array}{l}
u_{t}-a u_{x x x}+6 u_{x} u=2 b v v_{x} \\
v_{t}+v_{x x x}+3 u v_{x}=0
\end{array}\right.
$$

posed in $\mathbb{R}$ with $a \neq 0,-1$ and $b>0$. We prove that for $a \neq 0$, these systems are globally well-posed in $H_{p e r}^{1}([0, L]) \times H_{p e r}^{1}([0, L])$ by using the Bourgain's space framework. It is also showed the existence of a non-trivial smooth curve of periodic travelling waves solutions depending of the classical Jacobian elliptic function called cnoidal. Then we prove the non-linear stability of these waves solutions in the energy space $H_{p e r}^{1}([0, L]) \times H_{p e r}^{1}([0, L])$ in the case $a \geqq 1 / 20$. The Floquet theory is used to obtain a detailed spectral analysis of the Jacobian form of Lamé's equation which is required in our stability theory.
\end{abstract}

\section{Introduction}

In 1981, Hirota and Satsuma introduced the system of coupled Korteweg-de Vries equation $([9])$

$$
\left\{\begin{array}{l}
u_{t}-a\left(u_{x x x}+6 u_{x} u\right)=2 b v v_{x}, \quad x, t \in \mathbb{R} \\
v_{t}+v_{x x x}+3 u v_{x}=0,
\end{array}\right.
$$

*This work has been supported by CNPq Brazil under grant No. 300654/96-0 AMS Subject Classification: 76B25, 42B35, 42B05, 35Q53, 35Q51, 37K45, 35B10, 35A07. Key words and phrases. Dispersive equation, global well-posedness, Jacobian elliptic functions, nonlinear stability. 
for the real valued functions $u=u(x, t), v=v(x, t), a, b \in \mathbb{R}$. Equation (1.1) is usually referred as the Hirota-Satsuma systems. Equation (1.1) describes an interaction of two long waves with different dispersion relations. A lot of research on (1.1) has been conducted in the last two decades. For example, in [9] they showed that for all values of $a$ and $b$, system (1.1) possess the two conservation laws given in (1.3a)-(1.3b) below and they also found explicit solitary waves solutions with the classical secant hyperbolic profile. Moreover, for all values of $b$, but only $a=1 / 2$, this system is integrable and two more conserved quantities of higher order spatial derivative have been found ([7], [9], [10]).

Some previous works on the existence and non-linear stability of solitary wave solution has been obtained. Namely, existence of solutions of (1.1) of the form

$$
(u(x, t), v(x, t))=\left(\phi_{\lambda}(x-\lambda t), \psi_{\lambda}(x-\lambda t)\right)
$$

such that the profile $\left(\phi_{\lambda}, \psi_{\lambda}\right)$ has the infinite boundary condition $\phi_{\lambda}(\xi) \rightarrow$ $0, \psi_{\lambda}(\xi) \rightarrow 0$ as $|\xi| \rightarrow \infty$. Here, $\lambda$ represents the wave speed. The case $a=-1$, $b>0$ and $\phi_{\lambda}=k \psi_{\lambda}^{2}$ for some specific constant $k$, was studied in [12] where an explicit smooth family of solutions depending of parameter $\lambda>0$ was found. We remember that system (1.1) has two conserved quantities of motion,

$$
G(u, v)=\int\left[\frac{1+a}{2} u_{x}^{2}+b v_{x}^{2}-(1+a) u^{3}-b u v^{2}\right] d x
$$

and

$$
F(u, v)=\frac{1}{2} \int\left[u^{2}+\frac{2}{3} b v^{2}\right] d x .
$$

Now, since the solutions found in [12] are critical points of the functional

$$
B(u, v) \equiv G(u, v)+c F(u, v)
$$

namely, $B^{\prime}\left(\phi_{\lambda}, \psi_{\lambda}\right)=0$, then it is possible to apply the abstract stability theory development by Grillakis\&Shatah\&Strauss in [11] (see also Weinstein [20]) and obtain the non-linear stability of these solutions in $L^{2}(\mathbb{R}) \times H^{1}(\mathbb{R})$ by the flow generated by system (1.1).

We remember that the Grillakis et.al. theory establishes that a solitary wave solution or traveling wave solution $\left(\phi_{\lambda}, \psi_{\lambda}\right)$ is stable by the flow generated by 
equation (1.1) if it is a minimum of $G$ for a specific value of $F$. In fact, by supposing that $c \rightarrow\left(\phi_{\lambda(c)}, \psi_{\lambda(c)}\right)$ is a differentiable curve then we have that for every $c,\left(\phi_{\lambda(c)}, \psi_{\lambda(c)}\right)$ will be a local minimizer provided that

$$
d(c) \equiv G\left(\phi_{\lambda(c)}, \psi_{\lambda(c)}\right)+c F\left(\phi_{\lambda(c)}, \psi_{\lambda(c)}\right)
$$

is a strictly convex function of the parameter $c$, and the spectrum of the selfadjoint operator $B^{\prime \prime}\left(\phi_{\lambda(c)}, \psi_{\lambda(c)}\right)$ has exactly one negative eigenvalue which is simple, zero is a simple eigenvalue with eigenfunction $\left(\phi_{\lambda(c)}^{\prime}, \psi_{\lambda(c)}^{\prime}\right)$ and the remainder of the spectrum is bounded away from zero.

Recently, Lopes in [16], has considered the problem of the existence and stability of solitary wave solutions in the case $1+a>0$ and $b>0$ in (1.1). Via the Grillakis, et. al. theory and by using a spectral theory for self-adjoint ordinary differential operators given by $2 \times 2$ system, he shows that critical points of (1.3c) of the form $\left(\phi_{\lambda(c)}, 0\right)$ with $\lambda(c)=a c /(1+a)$ are stable if $a>-1 / 4$ and critical points of $(1.3 \mathrm{c})$ of the form $\left(k_{0} \psi_{\lambda(c)}^{2}, \psi_{\lambda(c)}\right)$ exists for $a<-1 / 4$, $\lambda(c)=c / 3>0$, and they are stable if $-1<a<-2 / 3$.

In a general form for nonlinear evolution equations under periodic boundary conditions, the problem of orbital stability has received little attention (see, by example, the recent works [1], [2] and [4]). Also, similar situation has occurred with the well-posedness issues. So, we intend to cast additional light on these issues, well-posedness and stability theory, in the particular case of the HirotaSatsuma systems (1.1) with $1+a>0$ and $a \neq 0$. As our general experience with evolution equations indicates that travelling waves when they exist, are often of fundamental importance in the development of a broad range of disturbance, we expect the issue for periodic waves solutions found by us will be interesting and important. Moreover, the existence and stability of travelling waves in the periodic case presents new points not encountered in case of the solitary waves.

We start by considering travelling wave solutions of (1.1) of the general form (1.2) with periodic conditions. So, $(\phi, \psi)$ satisfy the system

$$
\left\{\begin{array}{l}
-a\left(\phi^{\prime \prime}+3 \phi^{2}\right)-\lambda \phi=b \psi^{2}+D \\
\psi^{\prime \prime \prime}+3 \phi \psi^{\prime}-\lambda \psi^{\prime}=0
\end{array}\right.
$$


where $D$ is an integration constant. In the case $a+1>0, a \neq 0$ and $D=0$, we find solutions of the form $(\phi, 0)$ with $\lambda=\lambda(c)=-a c /(1+a)$. More precisely, we obtain a smooth curve $c \in\left(4 \pi^{2}(1+a) / L^{2},+\infty\right) \rightarrow\left(\phi_{c}, 0\right)$ where

$$
\phi_{c}(\xi)=\beta_{2}+\left(\beta_{3}-\beta_{2}\right) c n^{2}\left[\sqrt{\frac{\beta_{3}-\beta_{1}}{12}} \xi ; k\right],
$$

where $\operatorname{cn}(\cdot ; k)$ is the Jacobian elliptic function with modulus $k=k(c), \beta_{i}$ 's satisfying $\beta_{1}<0<\beta_{2}<\beta_{3}$ and $k^{2}=\frac{\beta_{3}-\beta_{2}}{\beta_{3}-\beta_{1}}$.

Next, since every solution $\left(\phi_{c}, 0\right)$ is a critical point for $B$ defined in (1.3c), by using Grillakis, et.al. theory, we show that these solutions are orbitally stable in $H_{p e r}^{1}([0, L]) \times H_{p e r}^{1}([0, L])$ with regard to the periodic flow of equation (1.1) provided that $a \geqq 1 / 20$. To show the spectral conditions required on the self-adjoint operator $B^{\prime \prime}\left(\phi_{c}, 0\right)$,

$$
B^{\prime \prime}\left(\phi_{c}, 0\right)=\left(\begin{array}{cc}
-(1+a) \frac{d^{2}}{d x^{2}}-6(1+a) \phi_{c}+c & 0 \\
0 & 2 b\left(-\frac{d^{2}}{d x^{2}}-\phi_{c}+\frac{c}{3}\right)
\end{array}\right)
$$

we need $a \geqq 1 / 20$ and the Floquet theory associated to the Jacobian form of Lamé's equation

$$
\frac{d^{2}}{d x^{2}} \Psi+\left[\rho-n(n+1) k^{2} s n^{2}(x ; k)\right] \Psi=0,
$$

with boundary conditions $\Psi(0)=\Psi(2 K), \Psi^{\prime}(0)=\Psi^{\prime}(2 K)$ and with specific values of $n$. Here $\operatorname{sn}(\cdot ; k)$ is the Jacobian elliptic function and $K=K(k)$ is the complete elliptic integral of the first kind. Now, the convexity condition of $d(c)$ is reduced to verify that the mapping $c \rightarrow F\left(\phi_{c}, 0\right)$ is a strictly increasing function. In this part of the analysis, the use of non-trivial relationship between the complete elliptic integrals of the first kind $K$ and the second kind $E$ are used (see Appendix for an explanation of the basic theory of elliptic integrals).

To complete our stability theory we require a theory of well-posedness for the initial value problem associated to (1.1) with $1+a>0$ and $a \neq 0$. So, by using the spaces of functions of space-time, $X_{s, b}$, introduced by Bourgain ([5]) (see (2.8) below), we will show that system (1.1) is locally well-posed in $H_{p e r}^{1}([0, L]) \times H_{p e r}^{1}([0, L])$ for any $a \neq 0$. We obtain this result via the 
contraction principle plus two basic nonlinear estimates. The first one is the bilinear estimate, $\left\|\eta(t) \partial_{x}(u v)\right\|_{X_{s,-\frac{1}{2}}} \leqq C\|u\|_{X_{s, \frac{1}{2}}}\|v\|_{X_{s, \frac{1}{2}}}, s \geqq 0$, and the second one is the novel estimate

$$
\left\|\eta^{2}(t) u \partial_{x} v\right\|_{X_{1,-\frac{1}{2}}} \leqq C\|u\|_{X_{0, \frac{1}{2}}}\|v\|_{X_{1, \frac{1}{2}}}
$$

for $\eta$ being a cut-off function. Finally, we obtain global well-posedness of (1.1) with initial data in $H_{p e r}^{1}([0, L]) \times H_{p e r}^{1}([0, L])$ for $1+a>0$ and using the conservation laws defined in (1.3a) and (1.3b).

We note that our stability result associated to solutions (1.5), are given for initial disturbances of the same period $L$. We conjecture that by disturbances of the type $n L, n \geqq 2$, these periodic travelling waves will be unstable.

In the case $a=-1$ the author have established in [3] a theory of stability in $L_{p e r}^{2}([0, L]) \times H_{p e r}^{1}([0, L])$ for the curve of dnoidal waves, $c \in\left(\frac{6 \pi^{2}}{L^{2}},+\infty\right) \rightarrow$ $\left(\phi_{c}, \psi_{c}\right)$, of (1.4) with $\lambda=\lambda(c)=c / 3$,

$$
\phi_{c}=b \psi_{c}^{2} / c, \quad \psi_{c}(\xi)=\sqrt{\frac{2 c}{3}-\eta_{2}^{2}} \sqrt{\frac{c}{b}} d n\left[\sqrt{\frac{c}{3}-\frac{\eta_{2}^{2}}{2}} \xi ; k\right],
$$

where $k$ and $\eta_{2}$ are depending smoothly on $c$.

The scheme of the paper is as follows: In Section 2 we present the estimates needed to establish our theory of local well-posedness for the system (1.1) in the case $a \neq 0$. In Section 3 we present how to construct the family of periodic travelling wave solutions defined in (1.5) with a fixed but arbitrary period $L>0$. In Section 4 we establish briefly the basic tools of the Floquet theory that we will use. Also, here we present a complete study of the spectrum associated to the Lamé's equation (1.7). Section 5 is devoted to establish the nonlinear stability theory associated to solutions $(1.5)$ in the norm $H_{p e r}^{1}([0, L]) \times H_{p e r}^{1}([0, L])$. Finally, in the Appendix we briefly review the basic definitions and properties of the complete elliptic integrals of first kind and Jacobi elliptic functions.

\section{Notation.}

The norm of a function $f \in L^{p}(\Omega)$ (equivalence class), for $\Omega$ an open subset of $\mathbb{R}$, is written $|f|_{L^{p}(\Omega)}^{p}=\int_{\Omega}|f|^{p} d x, p \geqq 1$. The inner product in $L^{2}(\Omega)$ of two 
functions $f, g$ is written as $(f, g)=\int_{\Omega} f \bar{g} d x$. To define the Sobolev spaces (of $L^{2}$ type) of periodic functions, we use the definitions and notions in Iorio\&Iorio [14]. Let $\mathcal{P}=C_{\text {per }}^{\infty}([0, L])=\left\{f: \mathbb{R} \rightarrow \mathbb{C} \mid f \in C^{\infty}(\mathbb{R})\right.$ and periodic with period $L>$ $0\}$. $\mathcal{P}^{\prime}$ (The topological dual of $\mathcal{P}$ ) is the collection of all continuous linear functionals from $\mathcal{P}$ into $\mathbb{C}$. $\mathcal{P}^{\prime}$ is called a set of periodic distributions. If $\Psi \in \mathcal{P}^{\prime}$ we denote the value of $\Psi$ at $\varphi$ by $\Psi(\varphi)=<\Psi, \varphi>$. Define the functions $\Theta_{k}(x)=$ $\exp (2 \pi i k x / L), k \in \mathbb{Z}, x \in \mathbb{R}$. The Fourier transform of $\Psi \in \mathcal{P}^{\prime}$ is the function

$\widehat{\Psi}: \mathbb{Z} \rightarrow \mathbb{C}$ defined by the formula $\left.\widehat{\Psi}(k)=\frac{1}{L}<\Psi, \Theta_{-k}\right\rangle, \quad k \in \mathbb{Z}$. So, if $\Psi$ is a periodic function with period $L$, for example $\Psi \in L^{2}(0, L)$, we have $\widehat{\Psi}(k)=$ $\frac{1}{L} \int_{0}^{L} \Psi(x) \exp (-2 \pi i k x / L) d x$. For $s \in \mathbb{R}$, the Sobolev space $H_{p e r}^{s}([0, L])$ is the set of all $f \in \mathcal{P}^{\prime}$ such that $\|f\|_{s}^{2}=L \sum_{k=-\infty}^{\infty}\left(1+|k|^{2}\right)^{s}|\widehat{f}(k)|^{2}<\infty$. $H_{\text {per }}^{s}([0, L])$ is a Hilbert space with respect to the inner product $(f \mid g)_{s}=L \sum_{k=-\infty}^{\infty}(1+$ $\left.|k|^{2}\right)^{s} \widehat{f}(k) \overline{\widehat{g}(k)}$. Let us remark that, since any function $f \in L^{2}([0, L])$ can be extended periodically, with period $L$, to all the real line, then we can identify isometrically $L^{2}([0, L])=H_{p e r}^{0}([0, L])$. So we have $(f \mid g)_{0}=(f, g)=\int_{0}^{L} f \bar{g} d x$ and $\|\cdot\|_{0}=\|\cdot\|$. Since for every $s \geqq 0 H_{p e r}^{s}([0, L]) \subset L_{p e r}^{2}([0, L])$, we have via Plancherel's identity that for every $n \in \mathbb{N},\|f\|_{n}^{2}=\sum_{j=0}^{n}\left\|f^{(j)}\right\|^{2}$, where $f^{(j)}$ represents the $j$-th derivative of $f$ taken in the sense of $\mathcal{P}^{\prime}$

\section{Global well-posedness theory in $H_{p e r}^{1}([0, L]) \times$ $H_{p e r}^{1}([0, L])$}

This section presents a result about the periodic global well-posedness problem to equation (1.1), with $1+a>0$, in $H_{p e r}^{1}([0, L]) \times H_{p e r}^{1}([0, L])$. The idea is to use the space-time spaces introduced by Bourgain ([5]) to obtain a local-well posedness theory in $H^{1}([0, L]) \times H_{p e r}^{1}([0, L])$ with $a \neq 0$. So from (1.3a)-(1.3b) we deduce our global result. Here we are not interested in the best Sobolev's exponent for which we obtain well-posedness but we are interested in a result which is sufficient to obtain our stability theory.

We start by introducing for a function $f=f(x, t)$ which is $L$-periodic with respect to the $x$ variable and $t \in \mathbb{R}$, the space-time Fourier transform $\hat{f}=\hat{f}(k, \tau)$ 
for $k \in \mathbb{Z}$ and $\tau \in \mathbb{R}$ by

$$
\hat{f}(k, \tau)=c \int_{-\infty}^{\infty} \int_{0}^{L} f(x, t) e^{-2 \pi i k x / L} e^{-i \tau t} d x d t .
$$

So, we have that $\hat{f}(k, \tau)=\left[\widehat{f}^{(x)}(k)\right]^{(t)}(\tau)$. This transform is inverted by

$$
f(x, t)=c \sum_{k \in \mathbb{Z}}\left[\int_{-\infty}^{\infty} \hat{f}(k, \tau) e^{i \tau t} d \tau\right] e^{2 \pi i k x / L} .
$$

So, it follows that $\widehat{f(t)}^{(x)}(k)=\int_{-\infty}^{\infty} \hat{f}(k, \tau) e^{i \tau t} d \tau$. The Fourier transform of the function $f g$ where $f=f(x, t)$ and $g=g(x, t)$ are $L$-periodic with respect to $x$ is obtained via convolution as

$$
\widehat{f g}(k, \tau)=\hat{f} \star \hat{g}(k, \tau)=c \sum_{m \in \mathbb{Z}} \int_{-\infty}^{\infty} \hat{f}(k-m, \tau-\gamma) \hat{g}(m, \gamma) d \gamma .
$$

Next, by using the Fourier inversion formula (2.2) we can write the solution of

$$
\left\{\begin{array}{l}
v \in C\left(\mathbb{R} ; L_{p e r}^{2}([0, L])\right), \\
v_{t}+v_{x x x}=0 \\
v(x, 0)=\phi(x)
\end{array}\right.
$$

denoted by $v(x, t) \equiv W(t) \phi(x)$, in the form

$$
v(x, t)=\sum_{n \in \mathbb{Z}} \hat{\phi}(n) e^{i n^{3} t} e^{2 \pi i n x / L} .
$$

We next find a Fourier transform notation for the solution of the linear inhomogeneous $L$-periodic initial value problem

$$
\left\{\begin{array}{l}
v_{t}+v_{x x x}=w, \quad x \in[0, L] \\
v(x, 0)=0,
\end{array}\right.
$$

with $w=w(x, t)$ a given $L$-periodic function in the variable $x$ and $t \in \mathbb{R}$. It is well-known via Duhamel's principle that

$$
v(x, t)=\int_{0}^{t} W(t-\tau) w(x, \tau) d \tau .
$$

So, from (2.4), relation $\int_{0}^{t} h\left(t^{\prime}\right) d t^{\prime}=\int_{-\infty}^{\infty} \frac{e^{i t \lambda}-1}{i \lambda} \hat{h}(\lambda) d \lambda$ and (2.1), we have

$$
v(x, t)=c \sum_{n \in \mathbb{Z}} e^{2 \pi i n x / L} \int_{-\infty}^{\infty} \hat{f}(n, \lambda) \frac{e^{i \lambda t}-e^{i n^{3} t}}{\lambda-n^{3}} d \lambda .
$$


To study the $L$-periodic initial value problem for the Hirota-Satsuma systems (1.1) with initial data $(\phi, \psi)$, we make the change of scale

$$
w(x, t)=u(\theta x, t), \text { with } \theta=-a^{1 / 3} .
$$

Then we obtain the system de equations

$$
\left\{\begin{array}{l}
w_{t}+w_{x x x}+6 a^{2 / 3} w_{x} w=-2 b a^{-1 / 3} V_{a} \partial_{x} V_{a}, \quad x \in\left[0, \theta^{-1} L\right] \\
v_{t}+v_{x x x}+3 W_{a} v_{x}=0, \quad x \in[0, L] \\
w(x, 0)=\phi(\theta x) \equiv \phi_{a}(x), v(x, 0)=\psi(x)
\end{array}\right.
$$

where $V_{a}(x, t)=v(\theta x, t)$ and $W_{a}(x, t)=w\left(\theta^{-1} x, t\right)$. So, we reduced our problem to prove well-posedness for system $(2.6)$ in $H_{p e r}^{1}\left(\left[0, \theta^{-1} L\right]\right) \times H_{p e r}^{1}([0, L])$. Here we will suppose without loss of generality that $a<0$. Then, for $\vec{w}=(w, v)$, $\vec{\phi}_{a}=\left(\phi_{a}, \psi\right)$, and $W(t) \vec{\phi}_{a}=\left(W(t) \phi_{a}, W(t) \psi\right)$, we need to find a continuous solution for the integral equation

$$
\vec{w}(t)=W(t) \vec{\phi}_{a}+\int_{0}^{t} W(t-\tau) \vec{F}(\tau) d \tau
$$

where $\vec{F}=(f, g) \equiv\left(-3 \theta^{2} \partial_{x}\left(w^{2}\right)+b \theta^{-1} \partial_{x}\left(V_{a}^{2}\right),-3 W_{a} v_{x}\right)$. Hence, from (2.4) and (2.5)

$$
\begin{aligned}
& w(t)=\sum_{n \in \mathbb{Z}} \hat{\phi}_{a}(n) e^{i\left(n^{3} t+2 \pi \theta n x / L\right)}+c \sum_{n \in \mathbb{Z}} e^{2 \pi \theta i n x / L} \int_{-\infty}^{\infty} \widehat{f}(n, \lambda) \frac{e^{i \lambda t}-e^{i n^{3} t}}{\lambda-n^{3}} d \lambda \\
& v(t)=\sum_{n \in \mathbb{Z}} \hat{\psi}(n) e^{i\left(n^{3} t+2 \pi n x / L\right)}+c \sum_{n \in \mathbb{Z}} e^{2 \pi i n x / L} \int_{-\infty}^{\infty} \widehat{g}(n, \lambda) \frac{e^{i \lambda t}-e^{i n^{3} t}}{\lambda-n^{3}} d \lambda .
\end{aligned}
$$

Equation (2.6a) will be solved using the contraction principle in the spaces $X_{s, b, \gamma}$. Here $X_{s, b, \gamma}$ for $s, b \in \mathbb{R}, \gamma>0$, is the closure of the Schwartz functions $\mathcal{S}([0, \gamma L] \times \mathbb{R})$, with respect to the norm

$$
\|u\|_{X_{s, b, \gamma}}=\left(\sum_{n \in \mathbb{Z}}(1+|n|)^{2 s} \int_{-\infty}^{\infty}\left(1+\left|\tau-n^{3}\right|\right)^{2 b}|\widehat{u}(n, \tau)|^{2} d \tau\right)^{1 / 2} .
$$

So, if we denote $<\sigma>=1+|\sigma|$ then $\|u\|_{X_{s, b, \gamma}}=\|<n>^{s}<\tau-n^{3}>^{b}$ $\widehat{u}(n, \tau) \|_{\ell^{2}\left(L_{\tau}^{2}\right)}$. Also we define the space $Y_{s, \gamma}, s \in \mathbb{R}$, as the closure of the Schwartz functions $\mathcal{S}([0, \gamma L] \times \mathbb{R})$, with respect to the norm

$$
\|u\|_{Y_{s, \gamma}}=\|u\|_{X_{s, \frac{1}{2}, \gamma}}+\left[\sum_{n \in \mathbb{Z}}(1+|n|)^{2 s}\left(\int_{-\infty}^{\infty}|\widehat{u}(n, \tau)| d \tau\right)^{2}\right]^{1 / 2} .
$$


So, $\|u\|_{Y_{s, \gamma}}=\|u\|_{X_{s, \frac{1}{2}, \gamma}}+\left\|<n>^{s} \widehat{u}(n, \tau)\right\|_{\ell^{2}\left(L_{\tau}^{1}\right)}$. Similarly, we introduce the spaces $Z_{s, \gamma}$ defined via the norm

$$
\|u\|_{Z_{s, \gamma}}=\|u\|_{X_{s,-\frac{1}{2}, \gamma}}+\left[\sum_{n \in \mathbb{Z}}(1+|n|)^{2 s}\left(\int_{-\infty}^{\infty} \frac{|\widehat{u}(n, \tau)|}{1+\left|\tau-n^{3}\right|} d \tau\right)^{2}\right]^{1 / 2} .
$$

Theorem 2.1. Let $\left(\phi_{a}, \psi\right) \in H_{p e r}^{1}\left(\left[0, \theta^{-1} L\right]\right) \times H_{p e r}^{1}([0, L])$ with $\widehat{\phi_{a}}(0)=\widehat{\psi}(0)=$ 0 . Then there exist $T=T\left(\left\|\left(\phi_{a}, \psi\right)\right\|_{H_{\text {per }}^{1} \times H_{\text {per }}^{1}}\right)>0$ and a unique solution $(w, v)$ of (2.6) with $(w(0), v(0))=\left(\phi_{a}, \psi\right)$ such that

$$
\begin{gathered}
(w, v) \in C\left([-T, T] ; H_{p e r}^{1}\left(\left[0, \theta^{-1} L\right]\right) \times H_{p e r}^{1}([0, L])\right) \cap\left(Y_{1, \theta^{-1}} \times Y_{1,1}\right), \\
\partial_{x}\left(w^{2}\right) \in X_{1, \frac{1}{2}, \theta^{-1}}, \quad \partial_{x}\left(v^{2}\right) \in X_{1, \frac{1}{2}, 1}, \quad \text { and }, \quad \eta(t) W_{a} \partial_{x} v \in X_{1, \frac{1}{2}, 1},
\end{gathered}
$$

for $\eta$ being a suitable cut-off function and $W_{a}(x, t)=w\left(\theta^{-1} x, t\right)$.

Moreover, for any $T^{\prime} \in(0, T)$ the map $\left(\phi_{a}, \psi\right) \rightarrow(w(t), v(t))$ is Lipschitz continuous from $H_{\text {per }}^{1}\left(\left[0, \theta^{-1} L\right]\right) \times H_{\text {per }}^{1}([0, L])$ to $C\left([-T, T] ; H_{\text {per }}^{1}\left(\left[0, \theta^{-1} L\right]\right) \times\right.$ $\left.H_{p e r}^{1}([0, L])\right) \cap\left(Y_{1, \theta^{-1}} \times Y_{1,1}\right)$.

Remark 2.2. The mean-zero assumption on the initial data $\left(\phi_{a}, \psi\right)$ is crucial for obtaining the main nonlinear estimates in subsection 2.2. For initial data with arbitrary mean value, we can use the arguments given by Bourgain in [5] (see also Staffilani [19]), therefore it will be omitted.

Remark 2.3. We note that the basic Bourgain-space $X_{s, \frac{1}{2}, \gamma}$ unfortunately does not give the properties of persistence and continuity of the solutions in the classical Sobolev spaces $H_{p e r}^{s}$, which is required in the classical definition of well-posedness and in our stability theory. We recall that for $b>1 / 2, X_{s, b} \subset$ $C\left(\mathbb{R}_{t} ; H_{\text {per }}^{s}\right)$. So, the additional norm in the R.H.S. of (2.9) appears as a sufficient condition for obtaining these two basic properties of our solutions found in $Y_{s, \gamma}$. In fact, inequality

$$
\sup _{t \in \mathbb{R}}\|u(t)\|_{H_{p e r}^{s}} \leqq\left[\sum_{n \in \mathbb{Z}}(1+|n|)^{2 s}\left(\int_{-\infty}^{\infty}|\widehat{u}(n, \tau)| d \tau\right)^{2}\right]^{1 / 2}
$$

shows the persistence property. Next, since $\int_{-\infty}^{\infty}|\widehat{u}(n, \tau)| d \tau<\infty$ for $n \in \mathbb{Z}$, we can ensure the continuity of the time flow of the solutions. Indeed, let $u \in Y_{s, \gamma}$ 
and define $g_{k}(\tau)=\hat{u}(k, \tau)$. Then we have that the inverse Fourier transform of $g_{k}$ in time exists. So,

$$
\|u(t+h)-u(t)\|_{H_{p e r}^{s}}^{2} \leqq \sum_{k \in \mathbb{Z}}<k>^{2 s}\left[\int_{-\infty}^{\infty}\left|g_{k}(\tau) \| e^{i h \tau}-1\right| d \tau\right]^{2} .
$$

Therefore, since

$$
<k>^{2 s}\left[\int_{-\infty}^{\infty}\left|g_{k}(\tau)\right|\left|e^{i h \tau}-1\right| d \tau\right]^{2} \leqq 2<k>^{2 s}\left[\int_{-\infty}^{\infty}\left|g_{k}(\tau)\right| d \tau\right]^{2} \equiv a_{k}
$$

and $\left\{a_{k}\right\} \in \ell^{1}(\mathbb{Z})$, it follows from the dominated convergence theorem that the flow $t \rightarrow u(t) \in H_{p e r}^{s}$ is continuous.

The result of continuous dependence in Theorem 2.1 permits regularization considerations, and so we can deduce formally that the quantities $G$ and $F$ in (1.3a) and (1.3b) respectively are conservations laws for equation (2.6). Similarly, we can show that the quantity

$$
H(z(t))=\int z(x, t) d x
$$

is other conservation law for (2.6). Therefore, using $G, F, 1+a>0$, and standard interpolations inequalities we deduce easily an a priori estimate for $\|(w, v)\|_{H_{p e r}^{1} \times H_{p e r}^{1}}$ and so we have the global well-posedness result for data in $H_{p e r}^{1}\left(\left[0, \theta^{-1} L\right]\right) \times H_{p e r}^{1}([0, L])$, that is,

Theorem 2.4. The solution obtained in Theorem 2.1 can be extended for any $T>0$ provided $1+a>0$.

The strategy for showing Theorem 2.1 is to find a solution of (2.6a) local in time in the space $Y_{1, \theta^{-1}} \times Y_{1,1}$. So, we consider a cut-off function $\varphi \in C_{0}^{\infty}(\mathbb{R})$ with $\varphi \equiv 1$ on $[-1,1]$ and supp $\varphi \subseteq[-2,2]$, then by using a fixed point argument we will construct a function $\vec{w}=(w, v) \in Y_{1, \theta^{-1}} \times Y_{1,1}$ satisfying

$$
\vec{w}(t)=\varphi(t) W(t) \vec{\phi}_{a}+\varphi(t) \int_{0}^{t} W(t-\tau) \vec{G}(\tau) d \tau
$$

where $\vec{G}(\tau)=\left(\varphi(\tau) f, \varphi^{2}(\tau) g\right)$ with $(f, g)=\left(-3 \theta^{2} \partial_{x}\left(w^{2}\right)+b \theta^{-1} \partial_{x}\left(V_{a}^{2}\right),-3 W_{a} v_{x}\right)$. Hence, for every $T \in(0,1]$ we find a solution $\vec{w}(t)$ of $(2.6 \mathrm{a})$ for $t \in[-T, T]$. 


\subsection{Linear estimates.}

In this subsection we obtain some basic estimates that are necessary in performing the contraction principle.

Lemma 2.5. For any $s \in \mathbb{R}$,

$$
\|\varphi(t) W(t) \zeta\|_{Y_{s, \gamma}} \leqq C\|\zeta\|_{H^{s}}
$$

Proof. Since $[\varphi W(t) \zeta]^{(n, \tau)}=\widehat{\zeta}^{(x)}(n) \widehat{\varphi}^{(t)}\left(\tau-n^{3}\right)$, we have

$$
\|\varphi(t) W(t) \zeta\|_{X_{s, \frac{1}{2}}}=\left\|<n>^{s}<\tau-n^{3}>^{1 / 2} \widehat{\zeta}(n) \widehat{\varphi}\left(\tau-n^{3}\right)\right\|_{\ell^{2}\left(L^{2}\right)}=\|\varphi\|_{H_{t}^{1 / 2}}\|\zeta\|_{H^{s}}
$$
and

$\left.\|<n>^{s}[\varphi(t) W(t) \zeta]^{(} n, \tau\right)\left\|_{\ell^{2}\left(L_{\tau}^{1}\right)}=\right\| \widehat{\varphi}\left\|_{L_{\tau}^{1}}\right\| \zeta \|_{H^{s}}$.

This finishes the Lemma.

Lemma 2.6. For any $s \in \mathbb{R}$ and $\vec{G}(\tau)=\left(\varphi(\tau) f, \varphi^{2}(\tau) g\right)$

$$
\left\|\varphi(t) \int_{0}^{t} W(t-\tau) \vec{G}(\tau) d \tau\right\|_{Y_{s, \gamma} \times Y_{s, 1}} \leqq C\|\vec{G}\|_{Z_{s, \gamma} \times Z_{s, 1}}
$$

Proof. The estimate for the norm in $X_{s, \frac{1}{2}, \gamma} \times X_{s, \frac{1}{2}, 1}$ has been already made in Bourgain [5], so we only establish this here. In fact, by using (2.5)-(2.7) we have the following

$$
\begin{aligned}
& \left\|\varphi(t) \int_{0}^{t} W(t-\tau) \vec{G}(\tau) d \tau\right\|_{X_{s, \frac{1}{2}, \gamma} \times X_{s, \frac{1}{2}, 1}} \\
& \leqq \\
& \quad C_{1}\left\|<n>^{s}<\tau-n^{3}>^{-1} \widehat{\vec{G}}(\tau)\right\|_{\ell^{2}\left(L_{\tau}^{1}\right) \times \ell^{2}\left(L_{\tau}^{1}\right)} \\
& \quad+C_{2}\left\|<n>^{s}<\tau-n^{3}>^{-1 / 2} \widehat{\vec{G}}(\tau)\right\|_{\ell^{2}\left(L_{\tau}^{2}\right) \times \ell^{2}\left(L_{\tau}^{2}\right)} \leqq C\|\vec{G}\|_{Z_{s, \gamma} \times Z_{s, 1}} .
\end{aligned}
$$

Next, we obtain the contribution of the second norm in the R.H.S. of (2.9) for the nonlinear part associated to $w$ in (2.7). In fact, for $\eta$ a smooth cut-off 
function in the time variable supported in $[-1,1]$ we have for $f_{0}=\varphi(\tau) f$

$$
\begin{aligned}
& \varphi(t) \int_{0}^{t} W(t-\tau) f_{0}(\tau) d \tau=\sum_{k \geqq 1} \frac{i^{k}}{k !} t^{k} \varphi(t)\left[\sum_{n \in \mathbb{Z}} e^{i\left(2 \pi \theta n x / L+n^{3} t\right)}\right. \\
& \left.\quad \int_{-\infty}^{\infty}\left(\lambda-n^{3}\right)^{k-1} \eta\left(\lambda-n^{3}\right) \widehat{f}_{0}(n, \lambda) d \lambda\right] \\
& +C \varphi(t) \sum_{n \in \mathbb{Z}} e^{2 \pi \theta i n x / L} \int_{-\infty}^{\infty}\left(\lambda-n^{3}\right)^{-1}(1-\eta)\left(\lambda-n^{3}\right) \widehat{f}_{0}(n, \lambda) e^{i \lambda t} d \lambda \\
& +C \varphi(t) \sum_{n \in \mathbb{Z}} e^{i\left(2 \pi \theta n x / L+n^{3} t\right)} \int_{-\infty}^{\infty}-\left(\lambda-n^{3}\right)^{-1}(1-\eta)\left(\lambda-n^{3}\right) \widehat{f_{0}}(n, \lambda) d \lambda \\
& \quad \equiv I+I I+I I I .
\end{aligned}
$$

Next, for $h_{k}(n)=\int_{-\infty}^{\infty} i^{k-1}\left(\lambda-n^{3}\right)^{k-1} \eta\left(\lambda-n^{3}\right) \widehat{f}_{0}(n, \lambda) d \lambda$ it follows immediately that $\left|h_{k}(n)\right| \leqq\left\|\chi_{[-1,1]}\left(\lambda-n^{3}\right) \widehat{f}_{0}(n, \lambda)\right\|_{L_{\tau}^{1}}$. So, for $\psi_{k}(t)=t^{k} \psi(t)$ we have that $\widehat{I}(n, \lambda)=\sum_{k \geqq 1} \frac{1}{k !} h_{k}(n) \widehat{\psi_{k}}\left(\lambda-n^{3}\right)$, and

$$
\begin{aligned}
\left\|<n>^{s} \widehat{I}(n, \lambda)\right\|_{\ell^{2}\left(L_{\lambda}^{1}\right)}^{2} & \leqq \sum_{n \in \mathbb{Z}}<n>^{2 s}\left(\sum_{k \geqq 1} \frac{1}{k !}\left\|\widehat{\psi}_{k}\right\|_{L_{\lambda}^{1}}\left\|\chi_{[-1,1]}\left(\lambda-n^{3}\right) \widehat{f}_{0}(n, \lambda)\right\|_{L_{\lambda}^{1}}\right)^{2} \\
& \leqq C\left\|<n>^{s}<\lambda-n^{3}>^{-1} \widehat{f}_{0}(n, \lambda)\right\|_{\ell^{2}\left(L_{\lambda}^{1}\right)}^{2} .
\end{aligned}
$$

Now, by denoting $\widehat{\zeta}(n)=-\int_{-\infty}^{\infty}\left(\lambda-n^{3}\right)^{-1}(1-\eta)\left(\lambda-n^{3}\right) \widehat{f}_{0}(n, \lambda) d \lambda$ we have that $I I I=\varphi(t) W(t) \zeta$ and so from Lemma 2.5 we have that

$$
\begin{aligned}
\left\|<n>^{s} \widehat{I I I}(n, \lambda)\right\|_{\ell^{2}\left(L_{\lambda}^{1}\right)}^{2} & \leqq C\|\zeta\|_{H_{x}^{s}}^{2} \\
& =C\left\|<n>^{s} \chi_{A}\left(\tau-n^{3}\right)\left|\tau-n^{3}\right|^{-1} \widehat{f}_{0}(n, \tau)\right\|_{\ell^{2}\left(L_{\tau}^{1}\right)}^{2} \\
& \leqq C\left\|<n>^{s}<\tau-n^{3}>^{-1} \widehat{f}_{0}(n, \tau)\right\|_{\ell^{2}\left(L_{\tau}^{1}\right)}^{2},
\end{aligned}
$$

where $\chi_{A}$ is the characteristic function of the set $A=\{\tau:|\tau| \geqq 1\}$.

Now we estimate $I I$. Let $l(n, \lambda)=\left(\lambda-n^{3}\right)^{-1}(1-\eta)\left(\lambda-n^{3}\right) \widehat{f}_{0}(n, \lambda)$. So from (2.2) it follows that $\widehat{I I}(k, \tau)=\left[\varphi(t) l^{\vee}(x, t)\right]^{(k, \tau)}=\widehat{\varphi} \star l(k, \tau)$. Then, by 
Young's inequality for convolutions we have

$$
\begin{aligned}
\left\|<n>^{s} \widehat{I I}(n, \tau)\right\|_{\ell^{2}\left(L_{\lambda}^{1}\right)}^{2} & =\sum_{n \in \mathbb{Z}}<n>^{2 s}\|\widehat{\varphi} \star l(n, \tau)\|_{L_{\tau}^{1}}^{2} \\
& \leqq \sum_{n \in \mathbb{Z}}<n>^{2 s}\|\widehat{\varphi}\|_{L_{\tau}^{1}}^{2}\|l(n, \tau)\|_{L_{\tau}^{1}}^{2} \\
& =C\left\|<n>^{s} \chi_{A}\left(\tau-n^{3}\right)\left|\tau-n^{3}\right|^{-1} \widehat{f}_{0}(n, \tau)\right\|_{\ell^{2}\left(L_{\tau}^{1}\right)}^{2} \\
& \leqq C\left\|<n>^{s}<\tau-n^{3}>^{-1} \widehat{f}_{0}(n, \tau)\right\|_{\ell^{2}\left(L_{\tau}^{1}\right)}^{2} .
\end{aligned}
$$

Therefore, from (2.14)-(2.17) we have

$$
\begin{aligned}
\|<n & >^{s}\left[\varphi(t) \int_{0}^{t} W(t-\tau) f_{0}(\tau) d \tau\right]^{(n, \tau)} \|_{\ell^{2}\left(L_{\tau}^{1}\right)} \\
& \leqq C\left\|<n>^{s}<\tau-n^{3}>^{-1} \widehat{f}_{0}(n, \tau)\right\|_{\ell^{2}\left(L_{\tau}^{1}\right)} \\
& \leqq\left\|f_{0}\right\|_{Z_{s, \gamma}} .
\end{aligned}
$$

Since a similar analysis is made for the nonlinear part of the function $v$ in (2.7), we obtain (2.13). This finishes the Lemma.

The following estimate established by Bourgain in [5] will be main tool in showing our nonlinear estimates .

Lemma 2.7. For functions on $\mathbf{T}^{2}$ we have

$$
\left\{\begin{array}{l}
\|f\|_{L^{4}\left(\mathbf{T}^{2}\right)} \leqq C\left(\sum_{m, n \in \mathbb{Z}}\left(1+\left|n-m^{3}\right|\right)^{2 / 3}|\widehat{f}(m, n)|^{2}\right)^{1 / 2} \\
\left(\sum_{m, n \in \mathbb{Z}}\left(1+\left|n-m^{3}\right|\right)^{-2 / 3}|\widehat{f}(m, n)|^{2}\right)^{1 / 2} \leqq C\|f\|_{L^{4 / 3}\left(\mathbf{T}^{2}\right)}
\end{array}\right.
$$

\subsection{Nonlinear estimates.}

In the next theorems $\eta=\eta(t)$ will represent a cut-off function supported in $[-1,1]$ say, such that $\widehat{\eta}$ is positive even Schwartz function. The proof of the following bilinear estimative was given by Bourgain in [5]. We denote $X_{s,-\frac{1}{2}, \gamma}$ by $X_{s,-\frac{1}{2}}$.

Theorem 2.8. For $s \geqq 0, u=u(x, t)$ and $v=v(x, t)$ periodic functions of $x$, and having zero $x$-mean for all $t$, we have

$$
\left\|\eta(t) \partial_{x}(u v)\right\|_{X_{s,-\frac{1}{2}}} \leqq C\|u\|_{X_{s, \frac{1}{2}}}\|v\|_{X_{s, \frac{1}{2}}}
$$


Remark 2.9. Extensions of inequality (2.18) for $s \in\left[-\frac{1}{2}, 0\right]$ can be found in Kenig, et. al. ([15]) and Colliander, et. al. ([8]).

Theorem 2.10. For $u=u(x, t)$ and $v=v(x, t)$ periodic functions of $x$, and having zero $x$-mean for all $t$, we have

$$
\left\|\eta^{2}(t) u \partial_{x} v\right\|_{X_{1,-\frac{1}{2}}} \leqq C\|u\|_{X_{0, \frac{1}{2}}}\|v\|_{X_{1, \frac{1}{2}}}
$$

Proof. By hypotheses we have that $\widehat{u}(0, \tau)=\widehat{v}(0, \tau)=0$ for all $\tau \in \mathbb{R}$. Initially,

$$
\widehat{\mid \eta^{2} u v_{x}}(n, \tau)\left|\leqq \int_{-\infty}^{\infty}\right| \widehat{u v_{x}}|(n, \tau-\lambda)| \widehat{\eta^{2}} \mid(\lambda) d \lambda
$$

Next,

$$
\left|\widehat{u v_{x}}\right|(n, p) \leqq \sum_{m \neq 0, n} \int_{-\infty}^{\infty}|\widehat{u}(m, \gamma)||n-m||\widehat{v}(n-m, p-\gamma)| d \gamma
$$

Define $c(n, \lambda)=<\lambda-n^{3}>^{1 / 2}|\widehat{u}(n, \lambda)|$ and $d(n, \lambda)=<n><\lambda-n^{3}>^{1 / 2}$ $|\widehat{v}(n, \lambda)|$. So,

$$
\begin{aligned}
& \frac{|n|\left|\widehat{u v_{x}}(n, p)\right|}{<\tau-n^{3}>^{1 / 2}} \\
& \quad \leqq \sum_{m \neq 0, n} \int_{-\infty}^{\infty} \frac{|n| c(m, \gamma) d(n-m, p-\gamma)}{<\tau-n^{3}>^{1 / 2}<\gamma-m^{3}>^{1 / 2}<p-\gamma-(n-m)^{3}>^{1 / 2}} d \gamma .
\end{aligned}
$$

Next, since $m \neq 0, m \neq n(n \neq 0)$, we obtain the well-known relation

$$
\left|\left(\tau-n^{3}\right)-\left[\left(\gamma-m^{3}\right)+\left(\tau-\gamma-(n-m)^{3}\right)\right]\right|=|3 m(n-m) n|>2 n^{2} .
$$

So, we have one of the following cases: $(i)\left|\tau-n^{3}\right|>\frac{1}{2} n^{2},(i i)\left|\gamma-m^{3}\right|>\frac{1}{2} n^{2}$, and $($ iii $)\left|\tau-\gamma-(n-m)^{3}\right|>\frac{1}{2} n^{2}$. Next, we consider each case separately:

Case (i). Define,

$$
\left\{\begin{array}{l}
F(x, t)=\sum_{m}\left[\int_{-\infty}^{\infty} \frac{c(m, \mu)}{<\mu-m^{3}>^{1 / 2}} e^{i \mu t} d \mu\right] e^{2 \pi i m x / L}, \\
G(x, t)=\sum_{m}\left[\int_{-\infty}^{\infty} \frac{d(m, \mu)}{<\mu-m^{3}>^{1 / 2}} e^{i \mu t} d \mu\right] e^{2 \pi i m x / L}
\end{array}\right.
$$


Then, since $\widehat{\eta}$ is a positive even Schwartz function, we have from (2.21) and

$$
\begin{aligned}
|n| \frac{\left|\widehat{\eta^{2} u v_{x}}(n, \tau)\right|}{<\tau-n^{3}>^{1 / 2}} & \leqq \sum_{m \neq 0, n} \int_{-\infty}^{\infty}\left[\widehat{F} \star_{\lambda} \widehat{\eta^{2}}\right](m, \gamma) \frac{d(n-m, \tau-\gamma)}{<\tau-\gamma-(n-m)^{3}>^{1 / 2}} d \gamma \\
& =\left[\widehat{F} \star_{\lambda} \widehat{\eta^{2}}\right] \star \widehat{G}(n, \tau)=\widehat{F G \eta^{2}}(n, \tau) .
\end{aligned}
$$

So, by using Plancherel and Cauchy-Schwarz

$$
\begin{aligned}
\left\|\eta^{2}(t) u \partial_{x} v\right\|_{X_{1,-\frac{1}{2}}} & \leqq\left[\sum_{n \neq 0} \int_{-\infty}^{\infty}\left|\widehat{F G \eta^{2}}(n, \tau)\right|^{2} d \tau\right]^{1 / 2}=C\left\|F G \eta^{2}\right\|_{L^{2}(d x \times d t)} \\
& \leqq C\|F\|_{L^{4}(d x \times d t(l o c))}\|G\|_{L^{4}(d x \times d t(l o c))} .
\end{aligned}
$$

Therefore, from Lemma 2.7 and (2.23) we have

$$
\|F\|_{L^{4}(d x \times d t(l o c))} \leqq C\left(\sum_{m} \int_{-\infty}^{\infty}|c(m, \lambda)|^{2} d \lambda\right)^{1 / 2}=C\|u\|_{X_{0, \frac{1}{2}}} .
$$

Similarly we show that $\|G\|_{L^{4}(d x \times d t(l o c))} \leqq C\|v\|_{X_{1, \frac{1}{2}}}$. This shows (2.19).

Case (ii). Let $H$ be such that $\widehat{H}(m, \mu)=c(m, \mu)$. Then from (2.21) and (2.23)

$$
\begin{aligned}
|n| \frac{\left|\widehat{\eta^{2} u v_{x}}(n, \tau)\right|}{<\tau-n^{3}>^{1 / 2}} & \leqq \sum_{m \neq 0, n} \int_{-\infty}^{\infty} \frac{c(m, \gamma)}{<\tau-n^{3}>^{1 / 2}}\left[\widehat{G} \star_{\lambda} \widehat{\eta^{2}}\right](n-m, \tau-\gamma) d \gamma \\
& =\frac{1}{<\tau-n^{3}>^{1 / 2}} \widehat{H} \star\left[\widehat{G} \star_{\lambda} \widehat{\eta^{2}}\right](n, \tau) \\
& =\frac{1}{<\tau-n^{3}>^{1 / 2}} \widehat{H G \eta^{2}}(n, \tau) .
\end{aligned}
$$

So, by using Plancherel, basic properties of convolutions and Lemma 2.7, we obtain

$$
\begin{aligned}
& \left\|\eta^{2}(t) u \partial_{x} v\right\|_{X_{1,-\frac{1}{2}}} \leqq\left[\sum_{n \neq 0} \int_{-\infty}^{\infty}\left|<\tau-n^{3}>^{-1 / 2} \widehat{H G \eta^{2}}(n, \tau)\right|^{2} d \tau\right]^{1 / 2} \\
& =\left(\sum_{n, m}<n-m^{3}>^{-1}|\widehat{H G}(m, n)|^{2}\right)^{1 / 2} \leqq C\|H G\|_{L^{4 / 3}(d x \times d t(l o c))} \\
& \quad \leqq C\|G\|_{L^{4}(d x \times d t(l o c))}\|H\|_{L^{2}(d x \times d t(l o c))} \leqq C\|v\|_{X_{1}, \frac{1}{2}}\|u\|_{X_{0}, \frac{1}{2}} .
\end{aligned}
$$

Case (iii) Let $J$ be such that $\widehat{J}(m, \mu)=d(m, \mu)$. Then from (2.21), (2.23) 
and Lemma 2.7 we obtain

$$
\begin{aligned}
& \left\|\eta^{2}(t) u \partial_{x} v\right\|_{X_{1,-\frac{1}{2}}} \leqq\left[\sum_{n \neq 0} \int_{-\infty}^{\infty}\left|<\tau-n^{3}>^{-1 / 2} \widehat{F J \eta^{2}}(n, \tau)\right|^{2} d \tau\right]^{1 / 2} \\
& =\left(\sum_{n, m}<n-m^{3}>^{-1}|\widehat{F J}(m, n)|^{2}\right)^{1 / 2} \leqq C\|F J\|_{L^{4 / 3}(d x \times d t(l o c))} \\
& \quad \leqq C\|F\|_{L^{4}(d x \times d t(l o c))}\|J\|_{L^{2}(d x \times d t(l o c))} \leqq C\|u\|_{X_{0}, \frac{1}{2}}\|v\|_{X_{1}, \frac{1}{2}}
\end{aligned}
$$

This finishes Theorem 2.10.

Remark 2.11. Inequality (2.19) can be extended in the following form. For $s \geqq 1, r \geqq s+n-1$ and $p-m \geqq s-1$, with $m \geqq 1$, we have

$$
\left\|\eta^{2}(t) \partial_{x}^{n} u \partial_{x}^{m} v\right\|_{X_{s,-\frac{1}{2}}} \leqq C\|u\|_{X_{r, \frac{1}{2}}}\|v\|_{X_{p, \frac{1}{2}}}
$$

The proof of the following estimate can be found in Bourgain [5].

Theorem 2.12. For $s \geqq 0, u=u(x, t)$ and $v=v(x, t)$ periodic functions of $x$ and having zero $x$-mean for all $t$, we have

$$
\left\|<k>^{s} \frac{\widehat{\eta(u v)_{x}}(k, \tau)}{<\tau-k^{3}>}\right\|_{\ell^{2}\left(L_{\tau}^{1}\right)} \leqq C\|u\|_{X_{s, \frac{1}{2}}}\|v\|_{X_{s, \frac{1}{2}}}
$$

Remark 2.13. Inequality (2.24) for $s=-1 / 2$ has been shown in Colliander et.al. [8].

Theorem 2.14. For $u=u(x, t)$ and $v=v(x, t)$ periodic functions of $x$ and having zero $x$-mean for all $t$, we have

$$
\left\|<k>\frac{\widehat{\eta^{2} u v_{x}}(k, \tau)}{<\tau-k^{3}>}\right\|_{\ell^{2}\left(L_{\tau}^{1}\right)} \leqq C\|u\|_{X_{0, \frac{1}{2}}}\|v\|_{X_{1, \frac{1}{2}}} .
$$

Proof. By using the same notation in the proof of Theorem 2.10 and the relation

$$
\frac{|n|\left|\widehat{u v_{x}}(n, p)\right|}{<\tau-n^{3}>} \leqq \sum_{m \neq 0, n} \int_{-\infty}^{\infty} \frac{|n| c(m, \gamma) d(n-m, p-\gamma)}{<\tau-n^{3}><\gamma-m^{3}>^{1 / 2}<p-\gamma-(n-m)^{3}>^{1 / 2}} d \gamma
$$


we have that the proof of the Theorem follows the same lines of proof of Lemma 7.42 in Bourgain [5] and so it will be omitted.

\subsection{Proof of Theorem 2.1.}

Initially we show that system (2.6) is well-posed with small initial data in the $H_{p e r}^{1}\left(\left[0, \theta^{-1} L\right]\right) \times H_{p e r}^{1}([0, L])$-norm. We denote by $\mathcal{V}_{\gamma}=\left\{h \in Y_{s, \gamma}: \int h(x, t) d x=\right.$ 0 , for all $t\}$. For $\vec{r}=(r, s) \in \mathcal{V}_{\theta^{-1}} \times \mathcal{V}_{1}$, we consider $f \equiv-3 \theta^{2} \partial_{x}\left(r^{2}\right)+$ $b \theta^{-1} \partial_{x}\left(S_{a}^{2}\right), g \equiv-3 R_{a} s_{x}$, with $S_{a}(x, t)=s(\theta x, t), R_{a}(x, t)=r\left(\theta^{-1} x, t\right)$, and $\vec{G}(\tau)=\left(\varphi(\tau) f, \varphi^{2}(\tau) g\right)$. Then, for $\vec{\phi}_{a}=\left(\phi_{a}, \psi\right) \in H_{p e r}^{1}\left(\left[0, \theta^{-1} L\right]\right) \times H_{p e r}^{1}([0, L])$ we define the operator

$$
\Phi_{\overrightarrow{\phi_{a}}}[\vec{r}(t)]=\varphi(t) W(t) \vec{\phi}_{a}+\varphi(t) \int_{0}^{t} W(t-\tau) \vec{G}(\tau) d \tau
$$

and the ball $\mathcal{B}=\left\{\vec{r} \in Y_{1, \theta^{-1}} \times Y_{1,1}:\|\vec{r}\|_{Y_{1, \theta^{-1}} \times Y_{1,1}} \leqq K\left\|\vec{\phi}_{a}\right\|_{H_{p e r}^{1} \times H_{p e r}^{1}}\right\} \cap \mathcal{V}_{\theta^{-1}} \times$ $V_{1}$. Next we show that $\Phi_{\overrightarrow{\phi_{a}}}$ is a contraction on $\mathcal{B}$ provided $\left\|\vec{\phi}_{a}\right\|_{H_{p e r}^{1} \times H_{p e r}^{1}}$ is sufficiently small. We first prove that $\Phi_{\vec{\phi}_{a}}$ sends bounded subset of $Y_{1, \theta^{-1}} \times Y_{1,1}$ in bounded subset of $Y_{1, \theta^{-1}} \times Y_{1,1}$. We estimate each scalar part of (2.26). For $\Phi_{\vec{\phi}_{a}}[\vec{r}(t)]=(p(t), q(t))$ we have from Lemma 2.5, Lemma 2.6, Theorem 2.8, and Theorem 2.12

$$
\begin{aligned}
\|p\|_{Y_{1, \theta^{-1}}} & \leqq C_{1}\left\|\phi_{a}\right\|_{1}+C_{2}\|\varphi(t) f\|_{Z_{1, \theta^{-1}}} \\
& \leqq C_{1}\left\|\phi_{a}\right\|_{1}+C_{2}\left(\|r\|_{X_{1, \frac{1}{2}, \theta^{-1}}}^{2}+\left\|S_{a}\right\|_{X_{1, \frac{1}{2}, \theta^{-1}}}^{2}\right) \\
& \leqq C_{1}\left\|\phi_{a}\right\|_{1}+C_{2}\left(\|r\|_{Y_{1, \theta^{-1}}}^{2}+\|s\|_{Y_{1,1}}^{2}\right) .
\end{aligned}
$$

where we have used that $\left\|S_{a}\right\|_{X_{1, \frac{1}{2}, \theta^{-1}}}^{2}=a^{-2 / 3}\|s\|_{X_{1, \frac{1}{2}, 1}}^{2}$. Similarly, from Lemma 2.5, Lemma 2.6, Theorem 2.10, and Theorem 2.14

$$
\begin{aligned}
\|q\|_{Y_{1,1}} \leqq & C_{1}\|\psi\|_{1}+C_{2}\left\|\varphi^{2}(t) g\right\|_{Z_{1,1}} \leqq C_{1}\|\psi\|_{1}+C_{2}\left(\left\|R_{a}\right\|_{X_{0, \frac{1}{2}, 1}}\|s\|_{X_{1, \frac{1}{2}, 1}}\right) \\
& \leqq C_{1}\|\psi\|_{1}+C_{2}\left(\|r\|_{Y_{1, \theta}-1}^{2}+\|s\|_{Y_{1,1}}^{2}\right)
\end{aligned}
$$

where $\left\|R_{a}\right\|_{X_{1, \frac{1}{2}, 1}}^{2}=a^{2 / 3}\|r\|_{X_{1, \frac{1}{2}, \theta^{-1}}}^{2}$. Therefore, we have

$$
\left\|\Phi_{\vec{\phi}_{a}}[\vec{r}(t)]\right\|_{Y_{1, \theta-1} \times Y_{1,1}} \leqq C_{1}\left\|\vec{\phi}_{a}\right\|_{H_{p e r}^{1} \times H_{p e r}^{1}}+C_{2}\|\vec{r}\|_{Y_{1, \theta}-1 \times Y_{1,1}}^{2}
$$

and the claim is proved. 
Now we prove that there is a $\beta \in(0,1)$ such that

$$
\left\|\Phi_{\vec{\phi}_{a}}[\vec{r}]-\Phi_{\vec{\phi}_{a}}[\vec{h}]\right\|_{Y_{1, \theta-1} \times Y_{1,1}} \leqq \beta\|\vec{r}-\vec{h}\|_{Y_{1, \theta}-1} \times Y_{1,1}
$$

for all $\vec{r}, \vec{h} \in \mathcal{B}$ provided that $\left\|\vec{\phi}_{a}\right\|_{H_{p e r}^{1} \times H_{p e r}^{1}}$ is sufficiently small. Let $\vec{h}=(j, h)$. Then since $j^{2}-r^{2}=(j-r)(j+r), S_{a}^{2}-H_{a}^{2}=\left(S_{a}-H_{a}\right)\left(S_{a}+H_{a}\right), R_{a} s_{x}-J_{a} h_{x}=$ $\left(R_{a}-J_{a}\right) s_{x}+J_{a}(s-h)_{x}$ we have from Lemma 2.6, Theorems 2.8-2.10-2.12 that

$$
\begin{aligned}
\left\|\Phi_{\vec{\phi}_{a}}[\vec{r}]-\Phi_{\vec{\phi}_{a}}[\vec{h}]\right\|_{Y_{1, \theta^{-1}} \times Y_{1,1}} & \leqq C_{3}\left(\|\vec{r}\|_{Y_{1, \theta^{-1}} \times Y_{1,1}}+\|\vec{h}\|_{Y_{1, \theta-1} \times Y_{1,1}}\right)\|\vec{r}-\vec{h}\|_{Y_{1, \theta^{-1}} \times Y_{1,1}} \\
& \leqq 2 C_{3} K\left\|\vec{\phi}_{a}\right\|_{H_{p e r}^{1} \times H_{p e r}^{1}}\|\vec{r}-\vec{h}\|_{Y_{1, \theta^{-1}} \times Y_{1,1}}
\end{aligned}
$$

Now, define $C=\max \left\{C_{1}, C_{2}, C_{3}\right\}$ and consider $K>2 C$. So, by choosing $\left\|\vec{\phi}_{a}\right\|_{H_{p e r}^{1} \times H_{p e r}^{1}}$ sufficiently small such that

$$
2 C K\left\|\vec{\phi}_{a}\right\|_{H_{p e r}^{1} \times H_{p e r}^{1}}<1
$$

it follows from $(2.27)$ and $(2.28)$ that $\Phi_{\vec{\phi}_{a}}(\mathcal{B}) \subseteq \mathcal{B}$ and $\Phi_{\vec{\phi}_{a}}$ is a contraction on $\mathcal{B}$. So, from (2.11) it follows that for $T \leqq 1$ there exists a curve $t \in[0, T] \rightarrow$ $\vec{w}(t)=(w, v)$ satisfying (2.6a). By Remark 2.3, $\vec{w} \in C\left([0, T] ; H_{p e r}^{1}\left(\left[0, \theta^{-1} T\right]\right) \times\right.$ $\left.H_{p e r}^{1}([0, T])\right)$ and satisfies (2.6) for any initial data satisfying (2.29). The property that this solution is unique requires a little more work. Since the proof of this follows the arguments in Staffilani [19] it will be omitted. The proof of the continuous dependence of the solution upon the data follows standard arguments (see [15]). Therefore, we obtain the Theorem 2.1 with the condition small on the initial data.

To remove the condition small on the initial data, we consider the following argument: We consider (2.6) with $L=L_{0}$ fixed and $\left(\phi_{a}, \psi\right) \in H_{p e r}^{1}\left(\left[0, \theta^{-1} L_{0}\right]\right) \times$ $H_{p e r}^{1}\left(\left[0, L_{0}\right]\right)$. This initial value problem is well-posed on a small time interval $[0, \delta]$ if and only if the $\lambda$-rescaled problem

$$
\left\{\begin{array}{l}
\partial_{t} w_{\lambda}+\partial_{x}^{3} w_{\lambda}+6 \theta^{2} w_{\lambda} \partial_{x} w_{\lambda}=2 b \theta^{-1} V_{a, \lambda} \partial_{x} V_{a, \lambda}, \quad x \in\left[0, \lambda \theta^{-1} L_{0}\right] \\
\partial_{t} v_{\lambda}+\partial_{x}^{3} v_{\lambda}+3 W_{a, \lambda} \partial_{x} v_{\lambda}=0, \quad x \in\left[0, \lambda L_{0}\right] \\
w_{\lambda}(x, 0)=\lambda^{2} \phi_{a}(x / \lambda) \equiv \phi_{a, \lambda}(x), \quad v_{\lambda}(x, 0)=\lambda^{2} \psi(x / \lambda) \equiv \psi_{\lambda}(x)
\end{array}\right.
$$

is well-posed on $\left[0, \lambda^{3} \delta\right]$. Next, for $I\left(\beta L_{0}\right) \equiv H_{p e r}^{1}\left(\left[0, \beta \theta^{-1} L_{0}\right]\right) \times H_{p e r}^{1}\left(\left[0, \beta L_{0}\right]\right)$ 
we have for $\lambda \geqq 1$ that

$$
\left\|\left(\phi_{a, \lambda}, \psi_{\lambda}\right)\right\|_{I\left(\lambda L_{0}\right)}^{2} \leqq \lambda^{-3}\left\|\vec{\phi}_{a}\right\|_{I\left(L_{0}\right)}^{2}
$$

Therefore by choosing $\lambda=\lambda\left(L_{0},\left\|\vec{\phi}_{a}\right\|_{I\left(L_{0}\right)}^{2}\right)$ sufficiently large, we have

$$
2 C K\left\|\left(\phi_{a, \lambda}, \psi_{\lambda}\right)\right\|_{I\left(\lambda L_{0}\right)} \leqq 2 C K \lambda^{-3 / 2}\left\|\vec{\phi}_{a}\right\|_{I\left(L_{0}\right)}<1 .
$$

This verifies (2.29) for the problem (2.30) and so we have well-posedness of (2.30) on the time interval, for example $[0,1]$. Therefore, $(2.6)$ is locally wellposed for $t \in\left[0, \lambda^{-3}\right]$. This finishes the proof.

Therefore, from Theorem 2.1, Theorem 2.4 and $u(x, t)=w\left(\theta^{-1} x, t\right)$, we obtain

Theorem 2.14. For $a \neq 0$ and $1+a>0$, system (1.1) is globally well-posed in $H_{p e r}^{1}([0, L]) \times H_{p e r}^{1}([0, L])$.

\section{Existence of cnoidal waves solutions.}

This section is devoted to establish the existence of a smooth curve of periodic travelling wave solutions to the Hirota-Satsuma systems (1.1) of the form

$$
\left\{\begin{array}{l}
u(x, t)=\phi(x-\lambda t) \\
v(x, t)=\psi(x-\lambda t)
\end{array}\right.
$$

when $b>0$ and $1+a>0($ with $a \neq 0)$. These solutions will be construct such that $(\phi, \psi)$ is a solution of the equation

$$
G^{\prime}(u, v)+c F^{\prime}(u, v)=0 .
$$

where $G$ and $F$ are defined in (1.3a)-(1.3b) and $c \in \mathbb{R}-\{0\}$ will belong to a specific interval. So, by substituting (3.1) in (1.1) and integrating once we obtain the non-linear system

$$
\left\{\begin{array}{l}
-a\left(\phi^{\prime \prime}+3 \phi^{2}\right)-\lambda \phi=b \psi^{2}+D \\
\psi^{\prime \prime \prime}+3 \phi \psi^{\prime}-\lambda \psi^{\prime}=0
\end{array}\right.
$$


where $D$ is an integration constant. Next we consider the existence of solutions for $(1.1)$ of the form $(u(x, t), v(x, t))=(\phi(x-\lambda t), 0)$ and $(u, v)$ satisfying $(3.2)$. So, we have that $\lambda=-a c /(1+a)$ and $D=0$ in (3.3). Therefore $\phi$ must satisfy

$$
\phi^{\prime \prime}+3 \phi^{2}-\frac{c}{1+a} \phi=0
$$

Next we show as to construct a smooth curve of solutions for (3.4) with a fixed fundamental period $L>0$ and depending of the parameter $c$. Initially, we make the change of variable $\varphi=6 \phi$ and so $\varphi$ must satisfy

$$
\varphi^{\prime \prime}+\frac{1}{2} \varphi^{2}-\frac{c}{1+a} \varphi=0
$$

Hence $\varphi$ satisfies the first-order equation

$$
\left[\varphi^{\prime}\right]^{2}=\frac{1}{3}\left[-\varphi^{3}+3 \frac{c}{1+a} \varphi^{2}+6 B_{\varphi}\right]=\frac{1}{3}\left(\varphi-\beta_{1}\right)\left(\varphi-\beta_{2}\right)\left(\beta_{3}-\varphi\right)
$$

where $\beta_{1}, \beta_{2}, \beta_{3}$ are the real zeros of the polynomial $F_{\varphi}(t)=-t^{3}+3 \frac{c}{1+a} t^{2}+6 B_{\varphi}$. Then we must to have the relations

$$
3 \frac{c}{1+a}=\beta_{1}+\beta_{2}+\beta_{3}, \quad 0=\sum_{i<j} \beta_{i} \beta_{j}, \quad 6 B_{\varphi}=\Pi_{i=1}^{3} \beta_{i} .
$$

We assume without losing generality that $\beta_{1}<\beta_{2}<\beta_{3}$. Now, from the first and second relations above we have for $\gamma \equiv \frac{c}{1+a}$ that

$$
-\beta_{1}=\frac{\beta_{2} \beta_{3}}{\beta_{2}+\beta_{3}}=\beta_{2}+\beta_{3}-3 \gamma
$$

and so $\beta_{2}, \beta_{3}$ belong to the rotated ellipse $\Xi(\gamma)$,

$$
\Xi(\gamma): \quad \beta_{2}^{2}+\beta_{3}^{2}+\beta_{2} \beta_{3}-3 \gamma\left(\beta_{2}+\beta_{3}\right)=0
$$

Then, since $\beta_{2}<\beta_{3}$ we have that $0<\beta_{2}<2 \gamma<\beta_{3}<3 \gamma$. Note that $\beta_{2} \leqq \varphi \leqq \beta_{3}$ and so $\varphi$ is a positive solution.

By defining $\zeta \equiv \varphi / \beta_{3}$, we have that (3.6) becomes

$$
\left[\zeta^{\prime}\right]^{2}=\frac{\beta_{3}}{3}\left(\zeta-\eta_{1}\right)\left(\zeta-\eta_{2}\right)(1-\zeta)
$$


where $\eta_{i}=\beta_{i} / \beta_{3}, i=0,1$. If we take the crest of the wave to be at $\xi=0, \zeta(0)=$ 1. Now, we define a further variable $\chi$ via the relation $\zeta=1+\left(\eta_{2}-1\right) \sin ^{2} \chi$, and so we get that

$$
\left(\chi^{\prime}\right)^{2}=\frac{\beta_{3}}{12}\left(1-\eta_{1}\right)\left[1-k^{2} \sin ^{2} \chi\right],
$$

where $k^{2}=\frac{1-\eta_{2}}{1-\eta_{1}}$. Note that $0<k^{2}<1$. So, for $l=\frac{\beta_{3}}{12}\left(1-\eta_{1}\right)$ we obtain that

$$
\int_{0}^{\chi(\xi)} \frac{d t}{\sqrt{1-k^{2} \sin ^{2} t}}=\sqrt{l} \xi
$$

It follows then from the definition of the Jacobian elliptic function $y=\operatorname{sn}(u ; k)$ (see Appendix) that $\sin \chi=\operatorname{sn}(\sqrt{l} \xi ; k)$ and hence

$$
\zeta=1+\left(\eta_{2}-1\right) \operatorname{sn}^{2}(\sqrt{l} \xi ; k) .
$$

Therefore, by using $\operatorname{sn}^{2} u+\operatorname{cn}^{2} u=1$, we arrive to the so-called cnoidal wave solution associated to equation (3.5)

$$
\varphi(\xi)=\varphi\left(\xi ; \beta_{1}, \beta_{2}, \beta_{3}\right)=\beta_{2}+\left(\beta_{3}-\beta_{2}\right) c n^{2}\left[\sqrt{\frac{\beta_{3}-\beta_{1}}{12}} \xi ; k\right],
$$

where $\beta_{i}$ 's satisfy (3.7) and $k^{2}=\frac{\beta_{3}-\beta_{2}}{\beta_{3}-\beta_{1}}$ (see [21]).

Next, since $\mathrm{cn}^{2}$ has fundamental period $2 K$ then $\varphi$ has fundamental period $T_{\varphi}$ given by

$$
T_{\varphi} \equiv \frac{4 \sqrt{3}}{\sqrt{\beta_{3}-\beta_{1}}} K(k) .
$$

Now we show that $T_{\varphi}>2 \pi / \sqrt{\gamma}$. Initially we express $T_{\varphi}$ as a function of $\beta_{2}$ and $\gamma$. In fact, for every $\beta_{2} \in(0,2 \gamma)$ there is a unique $\beta_{3} \in(2 \gamma, 3 \gamma)$ such $\left(\beta_{2}, \beta_{3}\right) \in \Xi(\gamma)$ where $2 \beta_{3}=3 \gamma-\beta_{2}+\sqrt{9 \gamma^{2}-3 \beta_{2}^{2}+6 \gamma \beta_{2}}$. So, by defining $\beta_{1} \equiv 3 \gamma-\beta_{2}-\beta_{3}$ we obtain for

$$
\begin{aligned}
& g\left(\beta_{2}, \gamma\right) \equiv \sqrt{9 \gamma^{2}-3 \beta_{2}^{2}+6 \gamma \beta_{2}}=\beta_{3}-\beta_{1}, \text { and } \\
& k^{2}\left(\beta_{2}, \gamma\right)=\frac{1}{2}+\frac{3\left(\gamma-\beta_{2}\right)}{2 g\left(\beta_{2}, \gamma\right)}
\end{aligned}
$$

that

$$
T_{\varphi}\left(\beta_{2}, \gamma\right)=\frac{4 \sqrt{3}}{\sqrt{g\left(\beta_{2}, \gamma\right)}} K\left(k\left(\beta_{2}, \gamma\right)\right)
$$


Then by fixing $\gamma>0$, we have $T_{\varphi}\left(\beta_{2}, \gamma\right) \rightarrow+\infty$, as $\beta_{2} \rightarrow 0$, and $T_{\varphi}\left(\beta_{2}, \gamma\right) \rightarrow$ $2 \pi / \sqrt{\gamma}$, as $\beta_{2} \rightarrow 2 \gamma$. So, since the mapping $\beta_{2} \in(0,2 \gamma) \rightarrow T_{\varphi}\left(\beta_{2}, \gamma\right)$ is strictly decreasing (see proof of Theorem 3.4) it follows that $T_{\varphi}>2 \pi / \sqrt{\gamma}$.

Now we obtain a cnoidal wave solution with period $L$. For $\gamma_{0}>4 \pi^{2} / L^{2}$ there is a unique $\beta_{2,0} \in\left(0,2 \gamma_{0}\right)$ such that $T_{\varphi}\left(\beta_{2,0}, \gamma_{0}\right)=L$. So, for $c_{0} \equiv(1+a) \gamma_{0}$ and $\beta_{3,0}$ such that $\left(\beta_{2,0}, \beta_{3,0}\right) \in \Xi\left(\gamma_{0}\right)$, we have that the cnoidal wave $\varphi(\cdot)=$ $\varphi\left(\cdot ; \beta_{1,0}, \beta_{2,0}, \beta_{3,0}\right)$ with $\beta_{1,0}=3 \gamma_{0}-\beta_{2,0}-\beta_{3,0}$, has fundamental period $L$ and satisfies (3.5) with $c=c_{0}$.

We note that by the analysis above we can see the cnoidal wave $\varphi\left(\cdot ; \beta_{1}, \beta_{2}, \beta_{3}\right)$ in (3.9) as a function depending only on $\gamma=c /(1+a)$ and $\beta_{2}$. So we will denote this dependence by $\varphi_{\gamma}\left(\cdot ; \beta_{2}\right)$ or $\varphi_{\gamma}$.

Next we show the existence of a smooth curve of cnoidal waves solutions for equation (3.4), in other words, we show that at least locally the choice of $\beta_{2,0}\left(\gamma_{0}\right)$ above depends smoothly of $\gamma_{0}$.

Theorem 3.1. Let $L>0$ be arbitrary but fixed. Consider $\gamma_{0}>\frac{4 \pi^{2}}{L^{2}}$ and the unique $\beta_{2,0} \in\left(0,2 \gamma_{0}\right)$ such that

$$
4 \sqrt{3} K\left(k\left(\beta_{2,0}, \gamma_{0}\right)\right)=L \sqrt{g\left(\beta_{2,0}, \gamma_{0}\right)} .
$$

Then,

(1) there exist an interval $J\left(\gamma_{0}\right)$ around of $\gamma_{0}$, an interval $B\left(\beta_{2,0}\right)$ around of $\beta_{2,0}$, and an unique smooth function $\Gamma: J\left(\gamma_{0}\right) \rightarrow B\left(\beta_{2,0}\right)$, such that $\Gamma\left(\gamma_{0}\right)=\beta_{2,0}$ and

$$
\frac{4 \sqrt{3}}{\sqrt{g\left(\beta_{2}, \gamma\right)}} K\left(k\left(\beta_{2}, \gamma\right)\right)=L
$$

where $\gamma \in J\left(\gamma_{0}\right), \beta_{2}=\Gamma(\gamma)$, and $k^{2}\left(\beta_{2}, \gamma\right), g\left(\beta_{2}, \gamma\right)$ are defined in (3.11).

(2) For $c \equiv(1+a) \gamma$ with $\gamma>4 \pi^{2} / L^{2}$, the cnoidal wave solution $\phi_{c}(\cdot)=$ $\frac{1}{6} \varphi_{\gamma}\left(\cdot ; \beta_{2}\right)$ has fundamental period $L$ and satisfies equation (3.4). Moreover, the mapping

$$
c \in\left(\frac{4 \pi^{2}}{L^{2}}(1+a),+\infty\right) \rightarrow \phi_{c} \in H_{p e r}^{n}([0, L])
$$

is a smooth function. 
Proof. The idea of the proof is to apply the implicit function theorem. We consider the open set $\Omega=\left\{\left(\beta_{2}, \gamma\right): \gamma>\frac{4 \pi^{2}}{L^{2}}, \beta_{2} \in(0,2 \gamma)\right\} \subseteq \mathbb{R}^{2}$ and define $\Phi: \Omega \rightarrow \mathbb{R}$ by

$$
\Phi\left(\beta_{2}, \gamma\right)=\frac{4 \sqrt{3}}{\sqrt{g\left(\beta_{2}, \gamma\right)}} K\left(k\left(\beta_{2}, \gamma\right)\right)-L
$$

where $g\left(\beta_{2}, \gamma\right)$ and $k^{2}\left(\beta_{2}, \gamma\right)$ are defined in (3.11). By hypotheses $\Phi\left(\beta_{2,0}, \gamma_{0}\right)=0$. Now we show that $\frac{\partial \Phi}{\partial \beta_{2}}\left(\beta_{2,0}, \gamma_{0}\right)<0$. In fact, by using the relations, $18 \gamma^{2}=$ $g^{2}\left(2-2 k^{2}+2 k^{4}\right)$,

$$
\frac{\partial g}{\partial \beta_{2}}=\frac{3\left(\gamma-\beta_{2}\right)}{g}, \quad \frac{\partial k}{\partial \beta_{2}}=-\frac{9 \gamma^{2}}{k g^{3}} \quad k k^{\prime 2} \frac{d K}{d k}=E-k^{\prime 2} K
$$

we have,

$$
\begin{aligned}
\frac{\partial \Phi}{\partial \beta_{2}}< & 0 \Leftrightarrow-18 \gamma^{2} \frac{d K}{d k}<g^{2}\left(2 k^{2}-1\right) k K \Leftrightarrow 18 \gamma^{2} E> \\
& \left(18 \gamma^{2}-k^{2}\left(2 k^{2}-1\right) g^{2}\right) k^{\prime 2} K \\
\Leftrightarrow & \left(2-2 k^{2}+2 k^{4}\right) E>\left(2-3 k^{2}+k^{4}\right) K .
\end{aligned}
$$

Next, since $E+K$ is a strictly increasing function we have that $\left(2-k^{2}\right) E>$ $2\left(1-k^{2}\right) K$. Moreover, from definition of the complete elliptical integrals $E$ and $K$ it follows that $\left(k^{2}-1\right) K \leqq\left(2 k^{2}-1\right) E$. So we obtain from (3.14) that $\frac{\partial \Phi}{\partial \beta_{2}}<0$.

Therefore, there is a unique smooth function, $\Gamma$, defined in a neighbourhood $J\left(\gamma_{0}\right)$ of $\gamma_{0}$, such that $\Phi(\Gamma(\gamma), \gamma)=0$ for every $\gamma \in J\left(\gamma_{0}\right)$. So, we obtain (3.12). Finally, since $\gamma_{0}$ was chosen arbitrarily in the interval $\mathcal{J}=\left(\frac{4 \pi^{2}}{L^{2}},+\infty\right)$, it follows that $\Gamma$ can extend to J. This completes the proof of the Theorem.

Corollary 3.2. It considers the mapping $\Gamma: J\left(\gamma_{0}\right) \rightarrow B\left(\beta_{2,0}\right)$ determined by Theorem 3.1. Then, $\beta_{2}(\gamma) \equiv \Gamma(\gamma)$ is a strictly decreasing function in $J\left(\gamma_{0}\right)$. Moreover, the modulus function

$$
k^{2}(\gamma)=\frac{1}{2}+\frac{3\left(\gamma-\beta_{2}(\gamma)\right)}{2 g\left(\beta_{2}(\gamma), \gamma\right)},
$$

where $g$ is defined by (3.11), it is a strictly increasing function. 
Proof. By Theorem 3.1 we have $\Phi(\Gamma(\gamma), \gamma)=0$ and so $\frac{d \Gamma}{d \gamma}=-\frac{\partial \Phi / \partial \gamma}{\partial \Phi / \partial \beta_{2}}$. Hence, we only need to show that $\partial \Phi / \partial \gamma<0$. In fact, from (3.11) and from relation $k g^{3} \frac{d k}{d \gamma}=9 \gamma \beta_{2}$ we have

$$
\frac{\partial \Phi}{\partial \gamma}<0 \Leftrightarrow 6 \gamma \beta_{2} \frac{d K}{d k}<g k\left(3 \gamma+\beta_{2}\right) K \Leftrightarrow 6 \gamma \beta_{2} E<\left[g k^{2}\left(3 \gamma+\beta_{2}\right)+6 \gamma \beta_{2}\right] k^{\prime 2} K .
$$

Now, since $g k^{2}\left(3 \gamma+\beta_{2}\right)+6 \gamma \beta_{2}=g^{2} / 2+g\left(3 \gamma+\beta_{2}\right) / 2$ implies $6 \gamma \beta_{2}<g\left(3 \gamma+\beta_{2}\right) k^{\prime 2}$, it follows from the inequality $E<K$ and (3.15) that $\frac{\partial \Phi}{\partial \gamma}<0$.

Finally, from definition of $k$ and $g$ we obtain $\frac{d k}{d \gamma}=\frac{9 \gamma}{k g^{3}}\left(\beta_{2}-\gamma \beta_{2}^{\prime}\right)>0$. This completes the proof.

\section{Spectral analysis.}

In this section we study the spectral properties associated to the linear operator $H_{a, c}=G^{\prime \prime}\left(\phi_{c}, 0\right)+c F^{\prime \prime}\left(\phi_{c}, 0\right)$ determined by the periodic solutions $\phi_{c}$ found in Theorem 3.1. So, we have the diagonal operator

$$
H_{a, c}=\left(\begin{array}{cc}
\mathcal{L}_{1} & 0 \\
0 & \mathcal{L}_{2}
\end{array}\right)
$$

where $\mathcal{L}_{1}=-(1+a) \frac{d^{2}}{d x^{2}}-6(1+a) \phi_{c}+c$ and $\mathcal{L}_{2}=2 b\left(-\frac{d^{2}}{d x^{2}}-\phi_{c}+\frac{c}{3}\right)$. We shall show that the spectrum of $H_{a, c}$ is discret and has its first two eigenvalues simple, being the eigenvalue zero the second one with eigenfunction $\left(\phi_{c}^{\prime}, 0\right)$ provided that $a \geqq 1 / 20$.

Initially, we have the following result about the periodic eigenvalue problem

$$
\left\{\begin{array}{l}
\mathcal{L}_{c n} \zeta \equiv\left(-\frac{d^{2}}{d x^{2}}-\varphi+\frac{c}{1+a}\right) \zeta=\lambda \zeta \\
\zeta(0)=\zeta(L), \quad \zeta^{\prime}(0)=\zeta^{\prime}(L)
\end{array}\right.
$$

where $\varphi$ is given by Theorem 3.1 .

Theorem 4.1. Let $c \in\left(\frac{4 \pi^{2}}{L^{2}}(1+a),+\infty\right)$ and $\varphi=\varphi_{c}$ be the cnoidal wave solution of (3.5) given by Theorem 3.1. Then, the linear operator $\mathcal{L}_{c n}$ in (4.2) defined on $H_{p e r}^{2}([0, L])$ has its first three eigenvalues simple, being the eigenvalue zero 
the second one with eigenfunction $\varphi^{\prime}$. Moreover, the remainder of the spectrum is constituted by a discret set of eigenvalues which are double.

Theorem 4.1 is a consequence of the Floquet theory (Magnus\&Winkler [17]). By convenience of the readers we will give some basic results of this theory. Initially, it follows from the Weyl's essential spectral theorem ([18]) that $\sigma_{\text {ess }}\left(\mathcal{L}_{c n}\right)=\sigma_{\text {ess }}\left(-\frac{d^{2}}{d x^{2}}+\frac{c}{1+a}\right)=\emptyset$. Therefore (4.2) determines a countably infinite set of eigenvalues $\left\{\lambda_{n} \mid n=0,1,2, \cdots\right\}$ with $\lambda_{0} \leqq \lambda_{1} \leqq \lambda_{2} \leqq \lambda_{3} \leqq \lambda_{4} \leqq \cdots$, where double eigenvalue is counted twice and $\lambda_{n} \rightarrow \infty$ as $n \rightarrow \infty$. We shall denote by $\zeta_{n}$ the eigenfunction associated to the eigenvalue $\lambda_{n}$. By the conditions $\zeta(0)=\zeta(L), \quad \zeta^{\prime}(0)=\zeta^{\prime}(L), \zeta_{n}$ can be extended to the whole of $(-\infty, \infty)$ as a continuously differentiable function with period $L$. Further, the double eigenvalues (if any) are the values of $\lambda$ for which all solutions of (4.2) have period $L$, that means the existence of two linearly independent periodic solutions of period $L$.

Now from Floquet theory, we know that the periodic eigenvalue problem (4.2) is related to the following semi-periodic eigenvalue problem considered on $[0, L]$

$$
\left\{\begin{array}{l}
\mathcal{L}_{c n} \xi=\mu \xi \\
\xi(0)=-\xi(L), \quad \xi^{\prime}(0)=-\xi^{\prime}(L)
\end{array}\right.
$$

which is also a self-adjoint problem and therefore determines a sequence of eigenvalues $\left\{\mu_{n} \mid n=0,1,2,3, \cdots\right\}$, with $\mu_{0} \leqq \mu_{1} \leqq \mu_{2} \leqq \mu_{3} \leqq \mu_{4} \leqq \cdots$, where double eigenvalue is counted twice and $\mu_{n} \rightarrow \infty$ as $n \rightarrow \infty$. We shall denote by $\xi_{n}$ the eigenfunction associated to the eigenvalue $\mu_{n}$. So, we have that the equation

$$
\mathcal{L}_{c n} f=\gamma f
$$

has a solution of period $L$ if and only if $\gamma=\lambda_{n}, n=0,1,2, \cdots$, as well as, it has a solution of period $2 L$ if and only if $\gamma=\mu_{n}, n=0,1,2, \cdots$. If all solutions of (4.4) are bounded we say that they are stable; otherwise we say that they are unstable. From the Oscillation Theorem we have that

$$
\lambda_{0}<\mu_{0} \leqq \mu_{1}<\lambda_{1} \leqq \lambda_{2}<\mu_{2} \leqq \mu_{3}<\lambda_{3} \leqq \lambda_{4} \cdots,
$$


and the intervals $\left(\lambda_{0}, \mu_{0}\right),\left(\mu_{1}, \lambda_{1}\right), \cdots$, are called intervals of stability. At the endpoints of these intervals the solutions of (4.4) are, in general unstable. This is always true for $\gamma=\lambda_{0}$ ( $\lambda_{0}$ is always simple). The intervals, $\left(-\infty, \lambda_{0}\right),\left(\mu_{0}, \mu_{1}\right),\left(\lambda_{1}, \lambda_{2}\right),\left(\mu_{2}, \mu_{3}\right), \cdots$, are called intervals of instability, omitting however any interval which is absent as a result of having a double eigenvalue. The interval of instability $\left(-\infty, \lambda_{0}\right)$ will always be present. We note that the absence of an instability interval means that there is a value of $\gamma$ for which all solutions of (4.4) have either period L or semi-period L-in other words, coexistence of solutions of (4.4) with period $L$ or period $2 L$ occurs for that value of $\gamma$.

We close this short review by establishing as is determined the number of zeros of $\zeta_{n}$ and $\xi_{n}$. Indeed,

(i) $\zeta_{0}$ has no zeros in $[0, L]$.

(ii) $\zeta_{2 n+1}$ and $\zeta_{2 n+2}$ have exactly $2 n+2$ zeros in $[0, L)$.

(iii) $\xi_{2 n}$ and $\xi_{2 n+1}$ have exactly $2 n+1$ zeros in $[0, L)$.

Proof.(Theorem 4.1) It follows from (4.5) that we need to show that $0=\lambda_{1}<$ $\lambda_{2}$. In fact, since $\mathcal{L}_{c n} \varphi^{\prime}=0$ and $\varphi^{\prime}$ has 2 zeros in $[0, L)$ then the eigenvalue 0 is either $\lambda_{1}$ or $\lambda_{2}$. We claim that $0=\lambda_{1}$. In fact, for $T_{\eta} \zeta(x) \equiv \zeta(\eta x)$ with $\eta^{2}=12 /\left(\beta_{3}-\beta_{1}\right)$ we have for $\Lambda \equiv T_{\eta} \zeta$ that

$$
\left\{\begin{array}{l}
\frac{d^{2}}{d x^{2}} \Lambda+\left[\rho-12 k^{2} s n^{2}(x)\right] \Lambda=0 \\
\Lambda(0)=\Lambda(2 K), \quad \Lambda^{\prime}(0)=\Lambda^{\prime}(2 K)
\end{array}\right.
$$

where for $\gamma=\frac{c}{1+a}$,

$$
\rho=-12\left[\gamma-\beta_{3}-\lambda\right] /\left(\beta_{3}-\beta_{1}\right) .
$$

The second order differential equation in (4.7) is called the Jacobian form of Lamé's equation. Now, from Floquet theory it follows that (4.7) has exactly 4 intervals of instability which are $\left(-\infty, \rho_{0}\right),\left(\mu_{0}^{\prime}, \mu_{1}^{\prime}\right),\left(\rho_{1}, \rho_{2}\right),\left(\mu_{2}^{\prime}, \mu_{3}^{\prime}\right)$ (where $\mu_{i}^{\prime}$, $i \geqq 0$, are the eigenvalues associated to the semi-periodic problem determined by Lamé's equation in (4.7)). Therefore, it follows that the first three eigenvalues $\rho_{0}, \rho_{1}, \rho_{2}$, associated to (4.7) are simple and the rest of eigenvalues $\rho_{3} \leqq \rho_{4}<$ $\rho_{5} \leqq \rho_{6}<\cdots$ satisfy that $\rho_{3}=\rho_{4}, \rho_{5}=\rho_{6}, \cdots$, in other words, they are double eigenvalues. 
For the sake of the exposition we will determine explicitly these eigenvalues. We start by noting that $\rho_{1}=4+4 k^{2}$ is an eigenvalue to (4.7) with eigenfunction $\Lambda_{1}(x)=\operatorname{cn}(x) \operatorname{sn}(x) d n(x)=k \cdot T_{\eta} \varphi^{\prime}(x)$, so $\lambda=0$ is a simple eigenvalue to (4.2) with eigenfunction $\varphi^{\prime}$. Now from Ince $([13])$ we have that the Lamé polynomials, $\Lambda_{0}(x)=d n(x)\left[1-\left(1+2 k^{2}-\sqrt{1-k^{2}+4 k^{4}}\right) \operatorname{sn}^{2}(x)\right]$ and $\Lambda_{2}(x)=d n(x)[1-(1+$ $\left.\left.2 k^{2}+\sqrt{1-k^{2}+4 k^{4}}\right) s n^{2}(x)\right]$, with period $2 K$, are the eigenfunctions associated to the others two eigenvalues $\rho_{0}, \rho_{2}$ given by

$$
\rho_{0}=2+5 k^{2}-2 \sqrt{1-k^{2}+4 k^{4}} \text { and } \rho_{2}=2+5 k^{2}+2 \sqrt{1-k^{2}+4 k^{4}} .
$$

Now, since $\Lambda_{0}$ has no zeros in $[0,2 K]$ and $\Lambda_{2}$ has exactly 2 zeros in $[0,2 K)$, it follows then that $\Lambda_{0}$ is the eigenfunction associated to $\rho_{0}$ which will be the first one eigenvalue to (4.7). Since $\rho_{0}<\rho_{1}$ for every $k^{2} \in(0,1)$, we obtain from the relation $-\beta_{1}\left(1+k^{2}\right)=\left(2-k^{2}\right) \beta_{3}-3 \gamma$, that

$$
12 \lambda_{0}=3 \frac{\beta_{3}-\gamma}{k^{2}+1} \rho_{0}+12\left(\gamma-\beta_{3}\right)<0
$$

and so $\lambda_{0}$ is the first negative eigenvalue to $\mathcal{L}_{c n}$ with eigenfunction $\zeta_{0}(x)=$ $\Lambda_{0}\left(\frac{1}{\eta} x\right)$. Now, since $\rho_{1}<\rho_{2}$ for every $k^{2} \in(0,1)$, we obtain that

$$
12 \lambda_{2}=3 \frac{\beta_{3}-\gamma}{k^{2}+1} \rho_{2}+12\left(\gamma-\beta_{3}\right)>0
$$

and so $\lambda_{2}$ is the third eigenvalue to $\mathcal{L}_{c n}$ with eigenfunction $\zeta_{2}(x)=\Lambda_{2}\left(\frac{1}{\eta} x\right)$.

Next, we can see that $\mu_{0}^{\prime}=5+2 k^{2}-2 \sqrt{4-k^{2}+k^{4}}$ and $\mu_{1}^{\prime}=5+5 k^{2}-$ $2 \sqrt{4-7 k^{2}+4 k^{4}}$ are the first two eigenvalues to Lamé's equation in the semiperiodic case, with eigenfunctions associated $\xi_{0, s m}(x)=c n(x)\left[1-\left(2+k^{2}-\right.\right.$ $\left.\left.\sqrt{4-k^{2}+k^{4}}\right) s n^{2}(x)\right]$ and $\xi_{1, s m}(x)=3 \operatorname{sn}(x)-\left(2+2 k^{2}-\sqrt{4-7 k^{2}+4 k^{4}}\right) s n^{3}(x)$ respectively. Since $\mu_{0}^{\prime}<\mu_{1}^{\prime}<4 k^{2}+4$ it follows from the relation

$$
\mu_{i}^{\prime}=-12 \frac{\gamma-\beta_{3}-\mu_{i}}{\beta_{3}-\beta_{1}}
$$

that the first three instability intervals associated to $\mathcal{L}_{c n}$ are $\left(-\infty, \lambda_{0}\right),\left(\mu_{0}, \mu_{1}\right)$, $\left(\lambda_{1}, \lambda_{2}\right)$. Finally, since the functions $\xi_{2, s m}(x)=c n(x)\left[1-\left(2+k^{2}+\sqrt{4-k^{2}+k^{4}}\right)\right.$ $\left.s n^{2}(x)\right]$ and $\xi_{3, s m}(x)=3 \operatorname{sn}(x)-\left(2+2 k^{2}+\sqrt{4-7 k^{2}+4 k^{4}}\right) s n^{3}(x)$ have three 
zeros in $[0,2 K)$ and are eigenfunctions of Lamé's equation in (4.7) with eigenvalues $\mu_{2}^{\prime}=5+2 k^{2}+2 \sqrt{4-k^{2}+k^{4}}$ and $\mu_{3}^{\prime}=5+5 k^{2}+2 \sqrt{4-7 k^{2}+4 k^{4}}$, it follows from (4.17) that the last instability interval of $\mathcal{L}_{c n}$ is $\left(\mu_{2}, \mu_{3}\right)$. This finishes the proof of the Theorem.

The following Lemma will be necessary in the study of the spectral structure of the linear operator $\mathcal{L}_{2}$ in $(4.1)$.

Lemma 4.2. The linear operator

$$
\mathcal{L}_{0} \zeta \equiv\left[-\frac{d^{2}}{d x^{2}}+2 k^{2} s n^{2}(x)\right] \zeta
$$

defined on $H_{\text {per }}^{2}([0,2 K])$ has $k^{2}$ as its first eigenvalue which is simple with eigenfunction $d n(x)$. The remainder of the spectrum is constituted by a discret set of eigenvalues which are double.

Proof. We consider the periodic eigenvalue problem on $[0,2 K]$

$$
\left\{\begin{array}{l}
\frac{d^{2}}{d x^{2}} \zeta+\left[\eta-2 k^{2} s n^{2}(x)\right] \zeta=0 \\
\zeta(0)=\zeta(2 K), \quad \zeta^{\prime}(0)=\zeta^{\prime}(2 K)
\end{array}\right.
$$

Then (4.10) determines a sequence of eigenvalues $\left\{\eta_{n}: n=0,1,2, \cdots\right\}$, with $\eta_{0} \leqq \eta_{1} \leqq \eta_{2} \leqq \cdots$. So, from Floquet theory it follows that (4.10) determines the existence of exactly 2 intervals of instability: $\left(-\infty, \eta_{0}\right),\left(\mu_{0}, \mu_{1}\right)\left(\mu_{i}\right.$ 's are the eigenvalues associated to the semi-periodic problem determined by $\mathcal{L}_{0}$ ). Moreover, it is easy to verify that $\eta_{0}=k^{2}$ is an eigenvalue with eigenfunction $d n$. This finishes the proof.

Theorem 4.3. Let $c \in\left(\frac{4 \pi^{2}}{L^{2}}(1+a),+\infty\right)$ and $\phi=\phi_{c}$ be the cnoidal wave solution of (3.4) given by Theorem 3.1. Then, the linear operator $\mathcal{L}_{2}$ in (4.1) is a strictly positive operator provided $a \geqq \frac{1}{20}$.

Proof. Let $\lambda \in \mathbb{R}$ and $g \in D\left(\mathcal{L}_{2}\right)$ such that $\mathcal{L}_{2} g=\lambda g$. So, for $\zeta(x)=g(p x)$ 
with $p=\sqrt{12 /\left(\beta_{3}-\beta_{1}\right)}$ and using the explicit form of $\phi$ we have that $\zeta$ satisfies

$$
-\zeta^{\prime \prime}+\left[\rho-2 k^{2} c n^{2}(x ; k)\right] \zeta=0, \quad \text { with } \rho=\frac{4 c-2 \beta_{2}-12 \lambda}{\beta_{3}-\beta_{1}} .
$$

So, from $s n^{2}+c n^{2}=1$, it follows from (4.11) that for $\mathcal{L}_{0}$ defined in Lemma 4.2,

$$
\mathcal{L}_{0} \zeta=\left[\frac{12 \lambda}{\beta_{3}-\beta_{1}}-\eta\right] \zeta, \quad \text { with } \eta=\frac{4 c-2 \beta_{2}}{\beta_{3}-\beta_{1}}-2 k^{2} .
$$

Then, from Lemma 4.2 it follows that

$$
\frac{12 \lambda}{\beta_{3}-\beta_{1}} \geqq k^{2}+\eta
$$

So, we have that $a \geqq \frac{1}{20}$ implies that $k^{2}+\eta>0$. In fact, from definition of $k^{2}$ and (3.7) we have

$$
k^{2}+\eta>0 \Leftrightarrow 4 c>\beta_{3}+\beta_{2} \Leftrightarrow \frac{(4 a+1) c}{1+a}>\frac{\beta_{2} \beta_{3}}{\beta_{2}+\beta_{3}} \equiv f\left(\beta_{2}, \beta_{3}\right) .
$$

Now the function $f$ defined in $[0,2 \gamma] \times[2 \gamma, 3 \gamma]$, with $\gamma=c /(1+a)$, assumes its maximum value, $6 \gamma / 5$, in the point $(2 \gamma, 3 \gamma)$. Therefore,

$$
\text { if } a \geqq \frac{1}{20} \Rightarrow \frac{(4 a+1) c}{1+a} \geqq \frac{6 c}{5(1+a)} .
$$

Therefore $\lambda>0$. This completes the Theorem.

Next we have the spectral structure required for $H_{a, c}$.

Theorem 4.4. Let $a \geqq 1 / 20, c \in\left(\frac{4 \pi^{2}}{L^{2}}(1+a),+\infty\right)$ and $\phi$ given by Theorem 3.1. Then the linear operator $H_{a, c}$ in (4.1) and defined on $H_{p e r}^{2}([0, L]) \times H_{p e r}^{2}([0, L])$ has exactly its first two eigenvalues simple, being the eigenvalue zero the second one with eigenfunction $\left(\phi^{\prime}, 0\right)$. Moreover, the remainder of the spectrum is constituted by a discret set of eigenvalues.

Proof. Let $(f, g) \in \operatorname{Ker}\left(H_{a, c}\right)$. Then $\mathcal{L}_{1} f=0$ and $\mathcal{L}_{2} g=0$. So, for $\varphi=6 \phi$ we have $\mathcal{L}_{c n} f=0$ and Theorem 4.1 implies that $f=\theta \phi^{\prime}$. Now, Theorem 4.3 implies that $g=0$ and so $(f, g)=\theta\left(\phi^{\prime}, 0\right)$. 
Next let $(f, g)$ and $\lambda<0$ be such that $H_{a, c}(f, g)^{t}=\lambda(f, g)^{t}$. Then $\mathcal{L}_{c n} f=$ $\frac{\lambda}{1+a} f$ and Theorem 4.1 implies that $\lambda=\lambda_{0}(1+a)$ and $f=\mu \zeta_{0}$ where $\lambda_{0}$ is the negative eigenvalue for $\mathcal{L}_{c n}$ with eigenfunction $\zeta_{0}$ associated. Now, since $\mathcal{L}_{2} g=\lambda g$ it follows from Theorem 4.3 that $g=0$. Therefore, $H_{a, c}$ has exactly a negative eigenvalue simple, $\lambda_{0}(1+a)$, with eigenfunction associated $\left(\zeta_{0}, 0\right)$. Finally, since $\mathcal{L}_{1}$ and $\mathcal{L}_{2}$ have a discret spectrum then $H_{a, c}$ as well. This proof the Theorem.

\section{Non-linear stability of cnoidal waves.}

In this section we establish the non-linear stability of the periodic travelling wave solutions, $\vec{\phi}_{c}=\left(\phi_{c}, 0\right)$, found in Theorem 3.1. To accomplish that, we apply the framework developed by Grillakis\&Shatah\&Strauss in [11]. Since Theorem 2.15 gives us the required information on the global-well posedness of (1.1) and Theorem 4.4 the information on the linear operator $H_{a, c}$, we only need to verify the convexity of the function

$$
d(c)=G\left(\phi_{c}, 0\right)+c F\left(\phi_{c}, 0\right) .
$$

This condition is equivalent to show that $c \rightarrow \frac{1}{2} \int_{0}^{L} \phi_{c}^{2}(\xi) d \xi$ is a strictly increasing function for $c \in\left(4 \pi^{2}(1+a) / L^{2},+\infty\right)$.

Initially, we specific our definition of stability. Let $\tau_{s}$ be translation by $s$, $\tau_{s} f(x)=f(x+s)$ for $x \in \mathbb{R}$. For $\vec{\phi}=\left(\phi_{c}, \psi_{c}\right)$ we define the $\vec{\phi}$-orbit, as $\Omega_{\vec{\phi}}=\{\vec{\phi}(\cdot+s): s \in \mathbb{R}\}$. For $\epsilon>0$ the tubular neighbourhood, $U_{\epsilon}$, around of $\Omega_{\vec{\phi}}$ in $X=H_{p e r}^{1}([0, L]) \times H_{p e r}^{1}([0, L])$ is defined as

$$
U_{\epsilon}=\left\{\vec{u} \in X: \inf _{s \in \mathbb{R}}\left\|\vec{u}-\tau_{s} \vec{\phi}\right\|_{X} \leqq \epsilon\right\}
$$

So, we have the following definition,

Definition (Orbital Stability). Let $\vec{\phi} \in X$ be a travelling wave solution to equation (3.3). We said that the orbit $\Omega_{\vec{\phi}}$ is orbitally stable in $X$ by the flow of equation (1.1) if for each $\epsilon>0$ there is a $\delta=\delta(\epsilon)>0$ such that if $\vec{u}_{0} \in X$ 
and $\inf _{r \in \mathbb{R}}\left\|\vec{u}_{0}-\tau_{r} \vec{\phi}\right\|_{X}<\delta$ then the solution $\vec{u}(t)$ of (1.1) with $\vec{u}(0)=\vec{u}_{0}$ exists globally and satisfies

$$
\sup _{t \in \mathbb{R}} \inf _{r \in \mathbb{R}}\left\|\vec{u}(t)-\tau_{r} \vec{\phi}\right\|_{X}<\epsilon .
$$

Otherwise, we said that $\Omega_{\vec{\phi}}$ is $X$-unstable.

Theorem 5.1 (Stability Theorem). Let $c \in\left(\frac{4 \pi^{2}}{L^{2}}(1+a),+\infty\right)$ and $\phi_{c}$ given by Theorem 3.1. Then $c \rightarrow\left\|\phi_{c}\right\|^{2}$ is a strictly increasing function. Therefore, for $\vec{\phi}_{c}=\left(\phi_{c}, 0\right)$ the orbit $\Omega_{\vec{\phi}}$ is orbitally stable in $H_{p e r}^{1}([0, L]) \times H_{p e r}^{1}([0, L])$ by the flow of the Hirota-Satsuma systems (1.1) provided $a \geqq 1 / 20$.

Proof. For $c \in\left(\frac{4 \pi^{2}}{L^{2}}(1+a),+\infty\right)$ define $\gamma(c)=c / 1+a$. Then we know that $\phi_{c}=\varphi_{\gamma(c)} / 6$ with $\varphi_{\gamma(c)}$ the cnoidal wave defined by (3.9). Hence, for $\mathcal{H}(\gamma)=$ $\gamma \int_{0}^{L} \varphi_{\gamma}(\xi) d \xi$ with $\gamma>4 \pi^{2} / L^{2}$, it follows from (3.5) that $d^{\prime}(c)=\mathcal{H}(\gamma(c)) / 36$. Therefore, $d^{\prime \prime}(c)=\frac{1}{36(1+a)} \frac{d}{d \gamma} \mathcal{H}(\gamma)$. Hence, we need to see that $\frac{d}{d \gamma} \mathcal{H}(\gamma)>0$. Initially we obtain an expression for $\mathcal{G}(\gamma)=\int_{0}^{L} \varphi_{\gamma}(\xi) d \xi$. In fact, from (3.9), (3.12) and using [6] we obtain

$$
\begin{aligned}
\int_{0}^{L} \varphi_{\gamma}(\xi) d \xi & =\beta_{2} L+\frac{2 \sqrt{3}\left(\beta_{3}-\beta_{2}\right)}{\sqrt{\beta_{3}-\beta_{1}}} \int_{0}^{2 K} c n^{2}(x ; k) d x \\
& =\beta_{2} L+4 \sqrt{3} \sqrt{\beta_{3}-\beta_{1}}\left[E-{k^{\prime}}^{2} K\right]=\beta_{2} L+\frac{48 K}{L}\left[E-k^{\prime 2} K\right] .
\end{aligned}
$$

Next, we express $\beta_{2}$ in function of $k$ and $K$. Initially we show that $18 \gamma^{2}=$ $2 g^{2}\left(1-k^{2}+k^{4}\right)$. Indeed, from (3.27) we have $g\left(2 k^{2}-1\right)=3\left(\gamma-\beta_{2}\right)$ and $g^{2}=9 \gamma^{2}-3 \beta_{2}^{2}+6 \gamma \beta_{2}$. Therefore, $g^{2}\left(2 k^{2}-1\right)^{2}+3 g^{2}=36 \gamma^{2}$ which shows our affirmation. So, by using (3.11) and (3.12) we obtain

$$
\beta_{2}=g\left(\frac{\gamma}{g}-\frac{2 k^{2}-1}{3}\right)=\frac{16 K^{2}}{L^{2}}\left[\sqrt{1-k^{2}+k^{4}}+1-2 k^{2}\right] .
$$

Then, from (5.1) it follows that

$$
\mathcal{G}(\gamma)=\frac{16 K^{2}}{L}\left[\sqrt{1-k^{2}+k^{4}}+k^{2}-2\right]+\frac{48}{L} K E \equiv \mathcal{J}(k(\gamma)) .
$$

Therefore, since $\mathcal{J}$ is a strictly increasing function of the parameter $k$ and from 
Corollary $3.2, k^{\prime}(\gamma)>0$, it follows that

$$
\frac{d}{d \gamma} \mathcal{H}(\gamma)=\mathcal{G}(\gamma)+\gamma \frac{d \mathcal{J}(k)}{d k} \frac{d k}{d \gamma}>0
$$

This completes the Theorem.

\section{Appendix}

In this Appendix we establish some basic properties about Jacobian elliptic functions (see Byrd\&Friedman [6]). Initially, we define the normal elliptic integral of the first kind

$$
\int_{0}^{y} \frac{d t}{\sqrt{\left(1-t^{2}\right)\left(1-k^{2} t^{2}\right)}}=\int_{0}^{\varphi} \frac{d \theta}{\sqrt{1-k^{2} \sin ^{2} \theta}} \equiv F(\varphi, k),
$$

where $y=\sin \varphi$, and the normal elliptic integral of the second kind,

$$
\int_{0}^{y} \sqrt{\frac{1-k^{2} t^{2}}{1-t^{2}}} d t=\int_{0}^{\varphi} \sqrt{1-k^{2} \sin ^{2} \theta} d \theta \equiv E(\varphi, k) .
$$

The number $k$ is called the modulus and belongs to the interval $(0,1)$. The number $k^{\prime}=\sqrt{1-k^{2}}$ is called the complementary modulus. $\varphi$ is called the argument of the normal elliptic integrals. It is usually understood that $0<y \leqq 1$ or $0<\varphi \leqq \pi / 2$. When $y=1$, the integrals above are said to be complete. In this case, one writes:

$$
\int_{0}^{1} \frac{d t}{\sqrt{\left(1-t^{2}\right)\left(1-k^{2} t^{2}\right)}}=\int_{0}^{\pi / 2} \frac{d \theta}{\sqrt{1-k^{2} \sin ^{2} \theta}}=F(\pi / 2, k) \equiv K(k) \equiv K,
$$

and

$$
\int_{0}^{1} \sqrt{\frac{1-k^{2} t^{2}}{1-t^{2}}} d t=\int_{0}^{\pi / 2} \sqrt{1-k^{2} \sin ^{2} \theta} d \theta=E(\pi / 2, k) \equiv E(k) \equiv E .
$$

So, $K(0)=E(0)=\pi / 2, E(1)=1$ and $K(1)=+\infty$. For $k \in(0,1), K^{\prime}(k)>0$, $K^{\prime \prime}(k)>0, E^{\prime}(k)<0$ and $E^{\prime \prime}(k)<0$. Moreover, for $k \in(0,1)$ we have that $E(k)<K(k)$, and $E(k)+K(k), E(k) K(k)$ are strictly increasing functions.

The complete elliptic integrals $K$ and $E$ satisfy the following hypergeometric differential equations

$$
\left\{\begin{array}{l}
k k^{\prime 2} \frac{d^{2} K}{d k^{2}}+\left(1-3 k^{2}\right) \frac{d K}{d k}-k K=0 \\
k k^{\prime 2} \frac{d^{2} E}{d k^{2}}+k^{\prime 2} \frac{d E}{d k}+k E=0
\end{array}\right.
$$


Now, we have some derivatives of the complete elliptical integrals $K$ and $E$,

$$
\left\{\begin{array}{l}
\frac{d K}{d k}=\frac{E-k^{\prime 2} K}{k k^{\prime 2}}, \quad \frac{d E}{d k}=\frac{E-K}{k}, \\
\frac{d^{2} E}{d k^{2}}=-\frac{1}{k} \frac{d K}{d k}=-\frac{E-k^{\prime 2} K}{k^{2} k^{\prime 2}} .
\end{array}\right.
$$

Next, we will define the Jacobian Elliptic Functions. Initially, we consider the elliptic integral

$$
u\left(y_{1} ; k\right) \equiv u=\int_{0}^{y_{1}} \frac{d t}{\sqrt{\left(1-t^{2}\right)\left(1-k^{2} t^{2}\right)}}=\int_{0}^{\varphi} \frac{d \theta}{\sqrt{1-k^{2} \sin ^{2} \theta}}=F(\varphi, k)
$$

which is a strictly increasing function of variable $y_{1}$ (real), hence we can define its inverse function by $y_{1}=\sin \varphi \equiv \operatorname{sn}(u ; k)$, or briefly $y_{1}=\operatorname{sn} u$, when it is not necessary to emphasize the modulus. The function $\operatorname{sn} u$ is a odd function. Other two basic functions can also be defined by

$$
\left\{\begin{array}{l}
\operatorname{cn}(u ; k)=\sqrt{1-y_{1}^{2}}=\sqrt{1-\operatorname{sn}^{2}(u ; k)} \\
\operatorname{dn}(u ; k)=\sqrt{1-k^{2} y_{1}^{2}}=\sqrt{1-k^{2} \operatorname{sn}^{2}(u ; k)}
\end{array}\right.
$$

requiring that $\operatorname{sn}(0, k)=0, \operatorname{cn}(0, k)=1$ and $\operatorname{dn}(0, k)=1$. The functions $\operatorname{cn} u$ and $\operatorname{dn} u$ are therefore even functions. The functions $\operatorname{sn}(u ; k), \operatorname{cn}(u ; k)$, and $\operatorname{dn}(u ; k)$ are called Jacobian elliptic functions and are one-valued functions of the argument $u$. These functions have a real period, namely, $4 K(k), 4 K(k)$ and $2 K(k)$ respectively. The more important properties of the Jacobian elliptic functions which have been used in this work are summarized by the following formulas:

$$
\operatorname{sn}^{2} u+\operatorname{cn}^{2} u=1, \quad k^{2} \operatorname{sn}^{2} u+\operatorname{dn}^{2} u=1, \quad k^{\prime 2} \operatorname{sn}^{2} u+\operatorname{cn}^{2} u=\operatorname{dn}^{2} u .
$$

Also, we have some special values:

$$
\left\{\begin{array}{l}
\operatorname{sn}(u+4 K)=\operatorname{sn} u, \quad \operatorname{cn}(u+4 K)=\operatorname{cn} u, \operatorname{dn}(u+2 K)=\operatorname{dn} u, \\
\operatorname{sn}(u+2 K)=-\operatorname{sn} u, \quad \operatorname{cn}(u+2 K)=-\operatorname{cn} u, \quad \operatorname{sn}(u, 0)=\sin u, \\
\operatorname{cn}(u, 0)=\cos u, \\
\operatorname{dn}(u, 0)=1, \quad \operatorname{sn}(u, 1)=\tanh u, \quad \operatorname{cn}(u, 1)=\operatorname{sech} u, \operatorname{dn}(u, 1)=\operatorname{sech} u .
\end{array}\right.
$$

Finally, we have the following differentiation formulas,

$$
\left\{\begin{array}{l}
\frac{\partial}{\partial u} \operatorname{sn} u=\operatorname{cn} u \operatorname{dn} u, \quad \frac{\partial}{\partial u} \operatorname{cn} u=-\operatorname{sn} u \operatorname{dn} u \\
\frac{\partial}{\partial u} \operatorname{dn} u=-k^{2} \operatorname{sn} u \operatorname{cn~} u .
\end{array}\right.
$$




\section{References}

[1] Alvarez, B.; Angulo, J., Existence and stability of perodic travelling-wave solutions of the Benjamin equation, To appear in Comm. on Pure and Appl. Analysis (2004).

[2] Angulo, J., Non-linear stability of periodic travelling-wave solutions to the Scrödinger and the modified Korteweg-de Vries, Pre-print (2003).

[3] Angulo, J., Stability of dnoidal waves to Hirota-Satsuma system, Pre-print (2004).

[4] Angulo, J.; Bona, J., Stability of Cnoidal waves, Pre-print (2003).

[5] Bourgain, J., Fourier restriction phenomena for certain lattice subsets and applications to nonlinear evolution equations, Geometric and Functional Anal., 3 (1993), 107-156, 209-262

[6] Byrd, P. F.; Friedman, M. D., Handbook of Elliptic Integrals for Engineers and Scientists, Sec. ed., Springer-Verlag, New York (1971).

[7] Chowdhury, Q. R.; Mukherjee, R., On the complete integrability of the Hirota-Satsuma system, J. Phys. A: Math. Gen., 17 (1977), 231-234.

[8] Colliander, J.; Keel, M.; Staffilani, G.; Takaoka, H.; Tao, T., Sharp global well-posedness for $K d V$ and modified $K d V$ on $\mathbb{R}$ and $\mathbf{T}$, J. Amer. Math. Soc., 16 (2003), 705-749.

[9] Hirota, R.; Satsuma, J., Soliton solution of a coupled KdV equations, Phys. Lett. A, 85 (1981), 407-409.

[10] Hirota, R.; Satsuma, J., A coupled KdV equation is one case of the fourreduction of the KP hierarchy, J. Phys. Soc. Jpn., 51 (1982), 3390-3397.

[11] Grillakis, M.; Shatah, J.; Strauss, H., Stability theory of solitary waves in the presence of symmetry I, J. Funct. Anal., 74 (1987), 160-197. 
[12] Gou, B.; Chen, L., Orbital stability of solitary waves of coupled KDV equations, Diff. Int. Equations, 3 (1999), 295-308.

[13] Ince, E. L., The periodic Lamé functions, Proc. Roy. Soc. Edin., 60 (1940), $47-63$.

[14] Iorio Jr., R. J.; Iorio de Magalhães, V., Fourier Analysis and Partial Differential Equations, Cambridge Stud. in Advan. Math., 70 (2001).

[15] Kenig, C.; Ponce, G.; Vega, L., A bilinear estimate with applications to the KdV equation, J. Amer. Math. Soc., 9 (1996), 573-603.

[16] Lopes, O., Stability of solitary waves of coupled systems, Pre-print (2003).

[17] Magnus, W., Winkler, S., Hill's Equation, Interscience, Tracts in Pure and Appl. Math. Wiley, NY., 20 (1976).

[18] Reed, S.; Simon, B., Methods of Modern Mathematical Physics: Analysis of Operator, Academic Press, V. iv (1978).

[19] Staffilani, G., On solutions for periodic generalized KdV equations, IMRN, 18 (1997), 899-917.

[20] Weinstein, M. I., Liapunov stability of ground states of nonlinear dispersive evolution equations, Comm. Pure Appl. Math., 39 (1986), 51-68.

[21] Whitam, G. B., Linear and Nonlinear waves, John Wiley, New York (1974).

Department of Mathematics

IMECC-UNICAMP

C.P. 6065

CEP 13083-970, Campinas, SP, Brazil

e-mail: angulo@ime.unicamp.br 